\title{
KAZHDAN-LUSZTIG CONJECTURE FOR GENERALIZED KAC-MOODY ALGEBRAS. II: PROOF OF THE CONJECTURE
}

\author{
SATOSHI NAITO
}

\begin{abstract}
Generalized Kac-Moody algebras were introduced by Borcherds in the study of Conway and Norton's moonshine conjectures for the Monster sporadic simple group. In this paper, we prove the Kazhdan-Lusztig conjecture for generalized Kac-Moody algebras under a certain mild condition, by using a generalization (to the case of generalized Kac-Moody algebras) of Jantzen's character sum formula. Our (main) formula generalizes the celebrated result for the case of Kac-Moody algebras, and describes the characters of irreducible highest weight modules over generalized Kac-Moody algebras in terms of the "extended" Kazhdan-Lusztig polynomials.
\end{abstract}

\section{INTRODUCTION}

This paper is the second part of our work on the Kazhdan-Lusztig conjecture for symmetrizable generalized Kac-Moody algebras. Here, as in our previous paper [N], a generalized Kac-Moody algebra (GKM algebra for short) is nothing but a complex contragredient Lie algebra $\mathfrak{g}(A)$ associated to a certain real square matrix (called a GGCM) $A=\left(a_{i j}\right)_{i, j \in I}$ indexed by a finite set $I=\{1,2, \ldots, n\}$ (see $[\mathrm{K}$, Chapter 11] and $\S 1$ below). (This definition is due to Kac, and slightly different from the one by Borcherds [B1].)

In [N], we proved that the multiplicity $\left[V((w, \beta) \circ \Lambda): L\left(\left(w^{\prime}, \beta^{\prime}\right) \circ \Lambda\right)\right]$ $\left((w, \beta),\left(w^{\prime}, \beta^{\prime}\right) \in W \times \mathscr{A}(\Lambda)\right)$ of the irreducible highest weight $\mathfrak{g}(A)$ module $L\left(\left(w^{\prime}, \beta^{\prime}\right) \circ \Lambda\right)$ with highest weight $\left(w^{\prime}, \beta^{\prime}\right) \circ \Lambda$ in the Verma module $V((w, \beta) \circ \Lambda)$ with highest weight $(w, \beta) \circ \Lambda$ is independent of the choice of the dominant integral weight $\Lambda \in P_{+}$. Furthermore, we conjectured that, under the condition on the GGCM $A=\left(a_{i j}\right)_{i, j \in I}$ that $a_{i i} \neq 0 \quad(i \in I)$, the multiplicity $\left[V((w, \beta) \circ \Lambda): L\left(\left(w^{\prime}, \beta^{\prime}\right) \circ \Lambda\right)\right]$ is equal to $P_{(w, \beta),\left(w^{\prime}, \beta^{\prime}\right)}(1)$, where $P_{(w, \beta),\left(w^{\prime}, \beta^{\prime}\right)}(q)$ is the extended Kazhdan-Lusztig polynomial in $q$ for $W \times \mathscr{A}$.

In the present paper, we show that this conjecture is true. Namely, we prove the following theorem.

Theorem I (Theorem 6.2.2). Let $\mathfrak{g}(A)$ be a GKM algebra. Assume that the GGCM $A=\left(a_{i j}\right)_{i, j \in I}$ is symmetrizable, and satisfies the condition that $a_{i i} \neq 0$

Received by the editors September 4, 1994.

1991 Mathematics Subject Classification. Primary 17B67, 17B65, 17B70; Secondary 17B10.

Key words and phrases. Generalized Kac-Moody algebra, character formula, Kazhdan-Lusztig conjecture.

Partially supported by Grant-in-Aid for Scientific Research (No. 05740015), The Ministry of Education, Science and Culture, Japan. 
$(i \in I)$. Then, for a dominant integral weight $\Lambda \in P_{+}$and $(w, \beta) \in W \times \mathscr{A}(\Lambda)$, we have

$$
\operatorname{ch} V((w, \beta) \circ \Lambda)=\sum_{\left(w^{\prime}, \beta^{\prime}\right) \in W \times \mathscr{A}(\Lambda)} P_{(w, \beta),\left(w^{\prime}, \beta^{\prime}\right)}(1) \operatorname{ch} L\left(\left(w^{\prime}, \beta^{\prime}\right) \circ \Lambda\right) .
$$

Equivalently, for $(w, \beta) \in W \times \mathscr{A}(\Lambda)$, we have

$$
\begin{aligned}
\operatorname{ch} L((w, \beta) \circ \Lambda)= & \sum_{\left(w^{\prime}, \beta^{\prime}\right) \in W \times \mathscr{A}(\Lambda)}(-1)^{\left(\ell\left(w^{\prime}\right)+\mathrm{ht}\left(\beta^{\prime}\right)\right)-(\ell(w)+\mathrm{ht}(\beta))} \\
\cdot & Q_{(w, \beta),\left(w^{\prime}, \beta^{\prime}\right)}(1) \operatorname{ch} V\left(\left(w^{\prime}, \beta^{\prime}\right) \circ \Lambda\right),
\end{aligned}
$$

where $Q_{(w, \beta),\left(w^{\prime}, \beta^{\prime}\right)}(q) \quad\left(\left(w^{\prime}, \beta^{\prime}\right) \in W \times \mathscr{A}(\Lambda)\right)$ are the extended inverse Kazhdan-Lusztig polynomials in $q$, such that for $(w, \beta),\left(w^{\prime}, \beta^{\prime}\right) \in W \times \mathscr{A}$,

$$
\begin{aligned}
& \sum_{(y, \gamma) \in W \times \mathscr{A}}(-1)^{(\ell(y)+\mathrm{ht}(\gamma))-(\ell(w)+\mathrm{ht}(\beta))} Q_{(w, \beta),(y, \gamma)}(q) P_{(y, \gamma),\left(w^{\prime}, \beta^{\prime}\right)}(q) \\
& \quad=\delta_{(w, \beta),\left(w^{\prime}, \beta^{\prime}\right) .}
\end{aligned}
$$

Here $\operatorname{ch} V$ denotes the formal character of a (weight) $\mathfrak{g}(A)$-module $V, \ell(w)$ is the length of $w \in W$, and $\mathrm{ht}(\beta)$ is the height of $\beta \in \mathscr{A}$.

Since GKM algebras obviously include Kac-Moody algebras, our result above is a generalization of the celebrated result (for Kac-Moody algebras) due to Kashiwara and Tanisaki [Ka, KT], or to Casian [C].

Our main tool is the following generalization (to symmetrizable GKM algebras) of Jantzen's character sum formula for a quotient of two Verma modules (cf. [J] and [RW]).

Theorem II (Theorem 4.3.12 and Proposition 5.2.1). Let $\mathfrak{g}(A)$ be a GKM algebra associated to a symmetrizable $G G C M A=\left(a_{i j}\right)_{i, j \in I}$ satisfying the condition that $a_{i i} \neq 0 \quad(i \in I)$. Let $\alpha=w\left(\alpha_{j}\right) \in W \cdot \Pi^{i m}$ with $w \in W$ and $\alpha_{j} \in \Pi^{i m}$, and let $\lambda \in \mathfrak{h}^{*}$ be such that $2(\lambda+\rho \mid \alpha)=(\alpha \mid \alpha)$. Then the quotient module $N(\lambda):=V(\lambda) / V(\lambda-\alpha)$ has a $\mathfrak{g}(A)$-module filtration

$$
N(\lambda)=N(\lambda)_{0} \supset N(\lambda)_{1} \supset N(\lambda)_{2} \supset \cdots
$$

such that

(1) $N(\lambda) / N(\lambda)_{1} \cong L(\lambda)$ as a $\mathfrak{g}(A)$-module,

(2) the following holds:

$$
\begin{aligned}
\sum_{i \geq 1} \operatorname{ch} N(\lambda)_{i}= & \sum_{\beta \in \Delta_{+}} \sum_{\substack{l \in \mathbb{Z}_{\geq 1} \\
2(\lambda+\rho \mid \beta)=l(\beta \mid \beta)}} \operatorname{ch} V(\lambda-l \beta) \\
& -\sum_{\gamma \in \Delta_{+}} \sum_{\substack{m \in \mathbb{Z}_{\geq 1} \\
2(\lambda-\alpha+\rho \mid \gamma)=m(\gamma \mid \gamma)}} \operatorname{ch} V(\lambda-\alpha-m \gamma)-a(\lambda) \operatorname{ch} V(\lambda-\alpha),
\end{aligned}
$$

where $a(\lambda) \in \mathbb{Z}$, the roots $\beta \in \Delta_{+}$and $\gamma \in \Delta_{+}$are taken with their multiplicities.

In particular, in the case where $\lambda=w(\Lambda+\rho)-\rho$ for $\Lambda \in P_{+}$with $\left(\Lambda \mid \alpha_{j}\right)=0$, the constant $a(\lambda)$ above is equal to 1 .

The proof of Jantzen's character sum formula for symmetrizable Kac-Moody algebras by Rocha-Caridi and Wallach [RW] can be adapted with only minor 
modifications to the case of symmetrizable GKM algebras $\mathfrak{g}(A)$ if we assume that the GGCM $A=\left(a_{i j}\right)_{i, j \in I}$ satisfies the condition that $a_{i i} \neq 0 \quad(i \in I)$. So we shall only sketch the proof, pointing out where the modifications are needed.

This paper is organized as follows. In $\S 1$ we recall some elementary facts about GKM algebras and the notation used in [N]. In $\S 2$ we review the notions of the extended Bruhat ordering on $W \times \mathscr{A}$ and the extended Kazhdan-Lusztig polynomials for $W \times \mathscr{A}$ introduced in [N]. In addition, we define the extended inverse Kazhdan-Lusztig polynomials for $W \times \mathscr{A}$. In $\S 3$, following [K] and [DGK], we give a brief account of fundamentals of the representation theory of GKM algebras that we need in this paper. In $\S 4$ we study two kinds of Jantzen's character sum formulas for symmetrizable GKM algebras. One corresponds to a single Verma module, and is proved more generally for symmetrizable contragredient Lie algebras by Kac and Kazhdan [KK]. The other corresponds to a quotient of two Verma modules, and plays an essential role in the proof of the Kazhdan-Lusztig conjecture (Theorems 6.1.2 and 6.2.2).

In $\S 5$, using the former character sum formula, we show that, for $\Lambda \in P_{+}$, $w \in W$, and $\alpha_{j} \in \mathscr{A}(\Lambda)$, the multiplicity $\left[V((w, 0) \circ \Lambda): L\left(\left(w, \alpha_{j}\right) \circ \Lambda\right)\right]$ is equal to $P_{w, w}(1)=1$ under the assumption that $a_{i i} \neq 0 \quad(i \in I)$. We then use this result to obtain further information ( $=$ the second assertion of Theorem II) about the latter character sum formula. In $\S 6$ we establish our main theorem (Theorem I) stated above.

I would like to express my sincere thanks to Professor Kiyokazu Suto for many valuable discussions and helpful suggestions.

\section{Preliminaries ANd Notation}

In this section, we recall some elementary facts about generalized Kac-Moody algebras from [B1] and [K, Chapter 11], and also fix notation.

1.1. Notation. Since this paper is a continuation of $[N]$, we follow its notation. Throughout this paper, all the vector spaces are over the field $\mathbb{C}$ of complex numbers, unless otherwise specifically stated. For a finite-dimensional vector space $V$, we denote by $\langle\cdot, \cdot\rangle$ a duality pairing between $V$ and its algebraic dual $V^{*}:=\operatorname{Hom}_{\mathbb{C}}(V, \mathbb{C})$.

For a Lie algebra $\mathfrak{a}, U(\mathfrak{a})$ denotes its universal enveloping algebra.

We denote by $\mathbb{R}$ the field of real numbers, and by $\mathbb{Z}$ the ring of rational integers. For an integer $m \in \mathbb{Z}$, we put

$$
\mathbb{Z}_{\geq m}:=\{k \in \mathbb{Z} \mid k \geq m\}, \quad \mathbb{Z}_{>m}:=\{k \in \mathbb{Z} \mid k>m\} .
$$

1.2. Generalized Kac-Moody algebras. Let $I=\{1,2, \ldots, n\}$ be a finite index set, and let $A=\left(a_{i j}\right)_{i, j \in I}$ be a real $n \times n$ matrix satisfying the following conditions:

(C1) either $a_{i i}=2$ or $a_{i i} \leq 0$ for $i \in I$;

(C2) $a_{i j} \leq 0$ if $i \neq j$, and $a_{i j} \in \mathbb{Z}$ for $j \neq i$ if $a_{i i}=2$;

(C3) $a_{i j}=0 \Leftrightarrow a_{j i}=0$.

We call such a matrix a GGCM (= generalized generalized Cartan matrix).

For any GGCM $A=\left(a_{i j}\right)_{i, j \in I}$, we have a triple $\left(\mathfrak{h}, \Pi=\left\{\alpha_{i}\right\}_{i \in I}, \Pi^{\vee}=\right.$ $\left.\left\{\alpha_{i}^{\vee}\right\}_{i \in I}\right)$ satisfying the following (see [K, Chapter 1]):

$(\mathrm{R} 1) \mathfrak{h}$ is a finite-dimensional (complex) vector space such that $\operatorname{dim}_{\mathbb{C}} \mathfrak{h}=$ $2 n-\operatorname{rank} A$ 
(R2) $\Pi=\left\{\alpha_{i}\right\}_{i \in I} \subset \mathfrak{h}^{*}$ is linearly independent, and $\Pi^{\vee}=\left\{\alpha_{i}^{\vee}\right\}_{i \in I} \subset \mathfrak{h}$ is linearly independent, where $\mathfrak{h}^{*}:=\operatorname{Hom}_{\mathbb{C}}(\mathfrak{h}, \mathbb{C})$;

(R3) $\left\langle\alpha_{j}, \alpha_{i}^{\vee}\right\rangle=a_{i j}(i, j \in I)$, where $\langle\cdot, \cdot\rangle$ denotes a duality pairing between $\mathfrak{h}$ and $\mathfrak{h}^{*}$.

The above triple is called a realization of $A$.

From now on, we assume that the GGCM $A=\left(a_{i j}\right)_{i, j \in I}$ is symmetrizable, i.e., there exists a diagonal matrix $D:=\operatorname{diag}\left(\varepsilon_{1}, \varepsilon_{2}, \ldots, \varepsilon_{n}\right)$ such that $\operatorname{det} D \neq$ 0 and $D^{-1} A$ is symmetric. Then we may and do assume that $\varepsilon_{i}>0$ for all $i \in I$.

The generalized Kac-Moody algebra (= GKM algebra) $\mathfrak{g}(A)$ associated to a symmetrizable GGCM $A=\left(a_{i j}\right)_{i, j \in I}$ is the Lie algebra (over $\mathbb{C}$ ) generated by the above vector space $\mathfrak{h}$ and the elements $e_{i}, f_{i}(i \in I)$ satisfying the following relations (see [B1], [K, Chapter 11]):

$$
\begin{aligned}
& \text { (F1) } \begin{cases}{\left[h, h^{\prime}\right]=0} & \text { for } h, h^{\prime} \in \mathfrak{h}, \\
{\left[h, e_{i}\right]=\left\langle\alpha_{i}, h\right\rangle e_{i}, \quad\left[h, f_{i}\right]=-\left\langle\alpha_{i}, h\right\rangle f_{i}} & \text { for } h \in \mathfrak{h}, i \in I, \\
{\left[e_{i}, f_{j}\right]=\delta_{i j} \alpha_{i}^{\vee}} & \text { for } i, j \in I,\end{cases} \\
& \text { (F2) }\left(\operatorname{ad} e_{i}\right)^{1-a_{i j} e_{j}=0,\left(\operatorname{ad} f_{i}\right)^{1-a_{i j}} f_{j}=0 \quad \text { if } a_{i i}=2 \text { and } j \neq i,}, \\
& \text { (F3) }\left[e_{i}, e_{j}\right]=0,\left[f_{i}, f_{j}\right]=0 \text { if } a_{i i}, a_{j j} \leq 0 \text { and } a_{i j}=0 .
\end{aligned}
$$

Then we have the root space decomposition of $\mathfrak{g}(A)$ with respect to the Cartan subalgebra h :

$$
\mathfrak{g}(A)=\mathfrak{h} \oplus \sum_{\alpha \in \Delta_{+}}^{\oplus} \mathfrak{g}_{\alpha} \oplus \sum_{\alpha \in \Delta_{-}}^{\oplus} \mathfrak{g}_{\alpha}
$$

where $\Delta_{+}\left(\subset Q_{+}:=\sum_{i \in I} \mathbb{Z}_{\geq 0} \alpha_{i}\right)$ is the set of positive roots, $\Delta_{-}\left(=-\Delta_{+}\right)$ is the set of negative roots, and $\mathfrak{g}_{\alpha}$ is the root space corresponding to a root $\alpha \in \Delta=\Delta_{+} \cup \Delta_{-} \subset \mathfrak{h}^{*}$. Note that $\mathfrak{g}_{\alpha_{i}}=\mathbb{C} e_{i}, \mathfrak{g}_{-\alpha_{i}}=\mathbb{C} f_{i}$ for $i \in I$, and that $\operatorname{mult}(\alpha):=\operatorname{dim}_{\mathbb{C}} \mathfrak{g}_{\alpha}=\operatorname{dim}_{\mathbb{C}} \mathfrak{g}_{-\alpha}<+\infty$ for $\alpha \in \Delta_{+}$.

Put $\mathfrak{n}_{+}:=\sum_{\alpha \in \Delta_{+}}^{\oplus} \mathfrak{g}_{\alpha}, \mathfrak{n}_{-}:=\sum_{\alpha \in \Delta_{+}}^{\oplus} \mathfrak{g}_{-\alpha}$, and $\mathfrak{b}:=\mathfrak{h} \oplus \mathfrak{n}_{+}$.

1.3. Imaginary simple roots. We put $I^{r e}:=\left\{i \in I \mid a_{i i}=2\right\}, I^{i m}:=\{i \in$ $\left.I \mid a_{i i} \leq 0\right\}$, and $\Pi^{r e}:=\left\{\alpha_{i} \in \Pi \mid i \in I^{r e}\right\}$ the set of real simple roots, $\Pi^{i m}:=\left\{\alpha_{i} \in \Pi \mid i \in I^{i m}\right\}$ the set of imaginary simple roots.

For $\alpha_{i}, \alpha_{j} \in \Pi^{i m}$, we say that $\alpha_{i}$ is perpendicular to $\alpha_{j}$ if $a_{i j}=0$. (Remark that an imaginary simple root $\alpha_{i} \in \Pi^{i m}$ is perpendicular to itself if $a_{i i}=0$.) For $\lambda \in \mathfrak{h}^{*}$ and $\alpha_{i} \in \Pi^{i m}$, we say that $\alpha_{i}$ is perpendicular to $\lambda$ if $\left\langle\lambda, \alpha_{i}^{\vee}\right\rangle=0$.

Now fix an element $\Lambda \in P_{+}:=\left\{\lambda \in \mathfrak{h}^{*} \mid\left\langle\lambda, \alpha_{i}^{\vee}\right\rangle \geq 0(i \in I)\right.$, and $\left\langle\lambda, \alpha_{i}^{\vee}\right\rangle \in$ $\mathbb{Z}_{\geq 0}$ if $\left.a_{i i}=2\right\}$. Then we define a subset $\mathscr{S}(\Lambda)$ (resp. $\mathscr{A}(\Lambda)$ ) of $\mathfrak{h}^{*}$ to be the set of all sums of distinct (resp. not necessarily distinct), pairwise perpendicular, imaginary simple roots perpendicular to $\Lambda$. Note that $\mathscr{A}:=\mathscr{A}(0)$ contains the set $\{0\} \cup \Pi^{i m} \cup\left\{m \alpha_{j} \mid m \in \mathbb{Z}_{\geq 2}, \alpha_{j} \in \Pi^{i m}\right.$ with $\left.a_{j j}=0\right\}$ by definition, while $m \alpha_{j}(m \geq 2)$ do not belong to $\mathscr{S}:=\mathscr{S}(0)$. For an element $\beta=\sum_{i \in I^{i m}} k_{i} \alpha_{i}$ $\left(k_{i} \in \mathbb{Z}_{\geq 0}\right)$, we put $\mathrm{ht}(\beta)=\sum_{i \in I^{i m}} k_{i}$, and call it the height of $\beta$.

1.4. Weyl group, real roots, and imaginary roots. For $i \in I^{r e}$, let $r_{i}$ be the simple reflection of $\mathfrak{h}^{*}$ given by: $r_{i}(\lambda)=\lambda-\left\langle\lambda, \alpha_{i}^{\vee}\right\rangle \alpha_{i} \quad\left(\lambda \in \mathfrak{h}^{*}\right)$. The Weyl group $W$ of $\mathfrak{g}(A)$ is the subgroup of $G L\left(\mathfrak{h}^{*}\right)$ generated by the $r_{i}$ 's $\left(i \in I^{r e}\right)$. Note that $\left(W,\left\{r_{i} \mid i \in I^{r e}\right\}\right)$ is a Coxeter system. For an element $w \in W$, $\ell(w)$ denotes the length of $w$. 
Let $\Delta^{r e}:=W \cdot \Pi^{r e}$ (the set of real roots), and $\Delta^{i m}:=\Delta \backslash \Delta^{r e}$ (the set of imaginary roots).

Here we recall the following characterization of positive imaginary roots: for an element $\alpha=\sum_{i \in I} k_{i} \alpha_{i} \in Q_{+} \backslash\{0\}$, we define $\operatorname{supp}(\alpha)$ to be the subdiagram of the Dynkin diagram of $A=\left(a_{i j}\right)_{i, j \in I}$ corresponding to the subset $\{i \in I \mid$ $\left.k_{i} \geq 1\right\}$ of $I$. Then we know that

$$
\Delta^{i m} \cap \Delta_{+}=\bigcup_{w \in W} w(K)
$$

where $K:=\left\{\alpha \in Q_{+} \backslash\{0\} \mid\left\langle\alpha, \alpha_{i}^{\vee}\right\rangle \leq 0(i \in I)\right.$, and $\operatorname{supp}(\alpha)$ is connected $\}$. In particular, the set $\Delta^{i m} \cap \Delta_{+}$of positive imaginary roots is $W$-stable.

For a real root $\alpha=w\left(\alpha_{i}\right) \quad\left(w \in W, \alpha_{i} \in \Pi^{r e}\right)$, we define the reflection $r_{\alpha}$ of $\mathfrak{h}^{*}$ with respect to $\alpha$ by: $r_{\alpha}(\lambda)=\lambda-\left\langle\lambda, \alpha^{\vee}\right\rangle \alpha \quad\left(\lambda \in \mathfrak{h}^{*}\right)$, where $\alpha^{\vee}:=$ $w\left(\alpha_{i}^{\vee}\right) \in \mathfrak{h}$ is the dual real root of $\alpha$. Note that $r_{\alpha}=w r_{i} w^{-1} \in W$.

1.5. Invariant bilinear forms. Since we have been assuming that the GGCM $A=\left(a_{i j}\right)_{i, j \in I}$ is symmetrizable, there exists a nondegenerate, symmetric, invariant bilinear form $(\cdot \mid \cdot)$ on $\mathfrak{g}(A)$. Note that the restriction of this bilinear form $(\cdot \mid \cdot)$ to the Cartan subalgebra $\mathfrak{h}$ is also nondegenerate, so that it induces (through a linear isomorphism $\nu: \mathfrak{h} \rightarrow \mathfrak{h}^{*}$ ) on $\mathfrak{h}^{*}$ a nondegenerate, symmetric, $W$-invariant bilinear form, which we again denote by $(\cdot \mid \cdot)$. Moreover, we have

$$
\nu\left(\alpha_{i}^{\vee}\right)=\varepsilon_{i} \alpha_{i} \quad(1 \leq i \leq n), \quad\left(\alpha_{i} \mid \alpha_{j}\right)=\varepsilon_{i}^{-1} \cdot a_{i j} \quad(1 \leq i, j \leq n) .
$$

We remark that a root $\alpha \in \Delta$ is an imaginary root if and only if $(\alpha \mid \alpha) \leq 0$.

1.6. Verma modules over GKM algebras and their irreducible quotients. For $\lambda \in \mathfrak{h}^{*}$, we denote by $V(\lambda)$ the Verma module $U(\mathfrak{g}(A)) \otimes_{U(\mathfrak{b})} \mathbb{C}(\lambda)$ with highest weight $\lambda$ over the GKM algebra $\mathfrak{g}(A)$. Here $\mathbb{C}(\lambda)$ is the one-dimensional $\mathfrak{h}$ module with weight $\lambda$, on which $\mathfrak{n}_{+}$acts trivially. As is well-known, the Verma module $V(\lambda)$ contains a unique maximal proper $\mathfrak{g}(A)$-submodule $V^{\prime}(\lambda)$. We define $L(\lambda)$ to be the quotient $\mathfrak{g}(A)$-module $V(\lambda) / V^{\prime}(\lambda)$, and hence $L(\lambda)$ is the irreducible highest weight $\mathfrak{g}(A)$-module with highest weight $\lambda$.

\section{BRUHAT ORDERING AND KAZHDAN-LUSZTIG POLYNOMIALS}

2.1. Extended Bruhat ordering. Here we recall the notion of the Bruhat ordering on the direct product $W \times \mathscr{A}$ of the Weyl group $W$ and $\mathscr{A}=\mathscr{A}(0)$ introduced in $[\mathrm{N}]$.

Definition 2.1.1 (Bruhat ordering). Let $w_{1}, w_{2} \in W$. We write $w_{1} \leftarrow w_{2}$ if there exists some $\gamma \in \Delta^{r e} \cap \Delta_{+}$such that $w_{1}=r_{\gamma} w_{2}$ and $\ell\left(w_{1}\right)=\ell\left(w_{2}\right)+1$. Moreover, for $w, w^{\prime} \in W$, we write $w \geqslant w^{\prime}$ if $w=w^{\prime}$ or if there exist $w_{1}, \ldots, w_{t} \in W$ such that

$$
w \leftarrow w_{1} \leftarrow \cdots \leftarrow w_{t} \leftarrow w^{\prime}
$$

Definition 2.1.2 ([N, Definition 2.3]). Let $\beta_{1}, \beta_{2} \in \mathscr{A}$. We write $\beta_{1} \leftarrow \beta_{2}$ if there exists some $\alpha_{j} \in \Pi^{i m}$ such that $\beta_{1}=\beta_{2}+\alpha_{j}$. Moreover, for $\beta=$ $\sum_{k \in I^{i m}} m_{k} \alpha_{k}, \beta^{\prime}=\sum_{k \in I^{i m}} m_{k}^{\prime} \alpha_{k} \in \mathscr{A}$, we write $\beta \geqslant \beta^{\prime}$ if $m_{k} \geq m_{k}^{\prime}$ for all $k \in I^{i m}$. 
Definition 2.1.3 ([N, Definition 2.4]). For $\left(w_{1}, \beta_{1}\right),\left(w_{2}, \beta_{2}\right) \in W \times \mathscr{A}$, we write $\left(w_{1}, \beta_{1}\right) \leftarrow\left(w_{2}, \beta_{2}\right)$

$$
\text { if } \quad w_{1} \leftarrow w_{2} \text { and } \beta_{1}=\beta_{2} \text { or if } \quad w_{1}=w_{2} \text { and } \beta_{1} \leftarrow \beta_{2} \text {. }
$$

Moreover, for $(w, \beta),\left(w^{\prime}, \beta^{\prime}\right) \in W \times \mathscr{A}$, we write $(w, \beta) \geqslant\left(w^{\prime}, \beta^{\prime}\right)$ if $w \geqslant w^{\prime}$ and $\beta \geqslant \beta^{\prime}$.

2.2. Extended Kazhdan-Lusztig polynomials. Let $W$ be the Weyl group of the GKM algebra $\mathfrak{g}(A)$. Recall that $\left(W,\left\{r_{i} \mid i \in I^{r e}\right\}\right)$ is a Coxeter system. Then the Hecke algebra $\mathscr{H}(W)$ of $W$ is the associative algebra over the Laurent polynomial ring $\mathbb{Z}\left[q^{\frac{1}{2}}, q^{-\frac{1}{2}}\right]$ (in the indeterminate $q^{\frac{1}{2}}$ ) which has a free $\mathbb{Z}\left[q^{\frac{1}{2}}, q^{-\frac{1}{2}}\right]$-basis $\left\{T_{w}\right\}_{w \in W}$ with the following relations:

(H1) $T_{w} T_{w^{\prime}}=T_{w w^{\prime}}$ if $\ell\left(w w^{\prime}\right)=\ell(w)+\ell\left(w^{\prime}\right) \quad\left(w, w^{\prime} \in W\right)$;

(H2) $\left(T_{r_{i}}+1\right)\left(T_{r_{i}}-q\right)=0 \quad\left(i \in I^{r e}\right)$.

Let $l$ be the involutive automorphism of the ring $\mathscr{H}(W)$ defined by: $l\left(q^{\frac{1}{2}}\right)=$ $q^{-\frac{1}{2}}, l\left(T_{w}\right)=\left(T_{w^{-1}}\right)^{-1} \quad(w \in W)$. Then we know the following proposition due to Kazhdan and Lusztig [KL1].

Proposition 2.2.1 ([KL1, Theorem 1.1]). For each $w \in W$, there exists a unique element $C_{w} \in \mathscr{H}(W)$ having the following properties:

(1) $l\left(C_{w}\right)=C_{w}$;

(2) $C_{w}=(-1)^{\ell(w)} q^{\frac{\ell(w)}{2}} \cdot \sum_{y \leqslant w}(-1)^{\ell(y)} q^{-\ell(y)} l\left(P_{y, w}(q)\right) T_{y}$, where $P_{w, w}(q)=$ 1 , and $P_{y, w}(q) \in \mathbb{Z}[q]$ is a polynomial with integer coefficients in the indeterminate $q$ of degree $\leq(1 / 2) \cdot(\ell(w)-\ell(y)-1)$ for $y \leq w, y \neq w$.

Moreover, the elements $C_{w} \quad(w \in W)$ form a free $\mathbb{Z}\left[q^{\frac{1}{2}}, q^{-\frac{1}{2}}\right]$-basis of $\mathscr{H}(W)$.

The above polynomials $P_{y, w}(q) \in \mathbb{Z}[q] \quad(y \leqslant w)$ are called the KazhdanLusztig polynomials. We set $P_{y, w}(q):=0$ unless $y \leqslant w$.

Now, for $\beta, \beta^{\prime} \in \mathscr{A}=\mathscr{A}(0)$, we define a polynomial $P_{\beta, \beta^{\prime}}(q)$ in $q$ by

$$
P_{\beta, \beta^{\prime}}(q):= \begin{cases}1 & \text { if } \beta^{\prime} \geqslant \beta \\ 0 & \text { otherwise. }\end{cases}
$$

Furthermore, for $(w, \beta),\left(w^{\prime}, \beta^{\prime}\right) \in W \times \mathscr{A}$, we put

$$
P_{(w, \beta),\left(w^{\prime}, \beta^{\prime}\right)}(q):=P_{w, w^{\prime}}(q) \cdot P_{\beta, \beta^{\prime}}(q) .
$$

We call the polynomials $P_{(w, \beta),\left(w^{\prime}, \beta^{\prime}\right)}(q)\left((w, \beta),\left(w^{\prime}, \beta^{\prime}\right) \in W \times \mathscr{A}\right)$ the extended Kazhdan-Lusztig polynomials for $W \times \mathscr{A}$.

It is also known (cf. [KL2], [KT]) that there exist the inverse Kazhdan-Lusztig polynomials $Q_{w, y}(q)(w \leqslant y \in W)$ for the Coxeter system $\left(W,\left\{r_{i} \mid i \in I^{r e}\right\}\right)$, such that

$$
\sum_{w \leqslant y \leqslant w^{\prime}}(-1)^{\ell(y)-\ell(w)} Q_{w, y}(q) P_{y, w^{\prime}}(q)=\delta_{w, w^{\prime}} \quad\left(w \leqslant w^{\prime}\right) .
$$

We set $Q_{w, y}(q):=0$ unless $w \leqslant y$.

We put $Q_{\beta, \beta^{\prime}}(q):=P_{\beta, \beta^{\prime}}(q)$ for $\beta, \beta^{\prime} \in \mathscr{A}$, and then

$$
Q_{(w, \beta),\left(w^{\prime}, \beta^{\prime}\right)}(q):=Q_{w, w^{\prime}}(q) \cdot Q_{\beta, \beta^{\prime}}(q)
$$


for $(w, \beta),\left(w^{\prime}, \beta^{\prime}\right) \in W \times \mathscr{A}$. We call the polynomials $Q_{(w, \beta),\left(w^{\prime}, \beta^{\prime}\right)}(q)$ $\left((w, \beta),\left(w^{\prime}, \beta^{\prime}\right) \in W \times \mathscr{A}\right)$ the extended inverse Kazhdan-Lusztig polynomials for $W \times \mathscr{A}$.

\section{BASIC REPRESENTATION THEORY OF GKM ALGEBRAS}

In this section, we briefly review some elementary notions and results of the representation theory of GKM algebras.

3.1. Category $\mathscr{O}$. Before defining the category $\mathscr{O}$, we introduce a partial ordering on $\mathfrak{h}^{*}$ by:

$$
\lambda \geq \mu\left(\lambda, \mu \in \mathfrak{h}^{*}\right) \quad \text { if } \quad \lambda-\mu \in Q_{+}=\sum_{i \in I} \mathbb{Z}_{\geq 0} \alpha_{i} .
$$

Now we define the category $\mathscr{O}$ of $\mathfrak{g}(A)$-modules as follows. The objects of $\mathscr{O}$ are $\mathfrak{g}(A)$-modules $V$ satisfying the following (see [K, Chapter 9]):

(1) $V$ admits a weight space decomposition

$$
V=\sum_{\tau \in \mathfrak{h}^{*}}^{\oplus} V_{\tau}
$$

with finite-dimensional weight spaces $V_{\tau}$;

(2) there exists a finite subset $\left\{\lambda_{1}, \ldots, \lambda_{s}\right\}$ of $\mathfrak{h}^{*}$ such that the set $P(V)$ of all weights of $V$ is contained in a finite union $\bigcup_{i=1}^{s} D\left(\lambda_{i}\right)$, where $D\left(\lambda_{i}\right):=\left\{\lambda \in \mathfrak{h}^{*} \mid \lambda \leq \lambda_{i}\right\} \quad(1 \leq i \leq s)$.

The morphisms in $\mathscr{O}$ are $\mathfrak{g}(A)$-module homomorphisms.

We note that the category $\mathscr{O}$ is closed under the operations of taking submodules, quotients, finite direct sums, and (finite) tensor products.

Obviously, highest weight $\mathfrak{g}(A)$-modules, such as $V(\lambda)$ and $L(\lambda) \quad\left(\lambda \in \mathfrak{h}^{*}\right)$, are in the category $\mathscr{O}$.

3.2. Formal characters and multiplicities. Here, from [K, Chapter 9] and [DGK], we recall the definitions of the formal character of a module $V$ in the category $\mathscr{O}$ and the multiplicity $[V: L(\mu)]$ of $L(\mu)$ in $V$ for $\mu \in \mathfrak{h}^{*}$.

First we define the algebra $\mathscr{E}$ as follows: the elements of $\mathscr{E}$ are series of the form $\sum_{\tau \in \mathfrak{h}^{*}} c_{\tau} e(\tau)$, where $c_{\tau} \in \mathbb{C}$ and $c_{\tau}=0$ for $\tau$ outside a finite union of sets of the form $D(\lambda)=\lambda-Q_{+}\left(\lambda \in \mathfrak{h}^{*}\right)$. Here the elements $e(\tau) \quad\left(\tau \in \mathfrak{h}^{*}\right)$ (called formal exponentials) are linearly independent, and are in one-to-one correspondence with the elements $\tau$ of $\mathfrak{h}^{*}$. The multiplication in $\mathscr{E}$ is defined by $e\left(\tau_{1}\right) \cdot e\left(\tau_{2}\right):=e\left(\tau_{1}+\tau_{2}\right) \quad\left(\tau_{1}, \tau_{2} \in \mathfrak{h}^{*}\right)$. Thus, $\mathscr{E}$ becomes a commutative associative algebra over $\mathbb{C}$ with the identity $e(0)$.

For a module $V=\sum_{\tau \in \mathfrak{h}^{*}}^{\oplus} V_{\tau}$ in the category $\mathscr{O}$, we define the formal character $\operatorname{ch} V$ of $V$ by $\operatorname{ch} V:=\sum_{\tau \in h^{*}}\left(\operatorname{dim}_{\mathbb{C}} V_{\tau}\right) e(\tau)$. Clearly, we have $\operatorname{ch} V \in \mathscr{E}$ for a module $V$ in the category $\mathscr{O}$.

Furthermore, a family $\left\{f_{x}=\sum_{\tau \in \mathfrak{h}^{*}} c_{x \tau} e(\tau)\right\}_{x \in X}$ of elements in $\mathscr{E}$ indexed by an arbitrary set $X$ is said to be summable if

(1) there exist $\lambda_{1}, \ldots, \lambda_{k} \in \mathfrak{h}^{*}$ such that, for each $x \in X, c_{x \tau}=0$ for $\tau$ outside $\bigcup_{i=1}^{k} D\left(\lambda_{i}\right)$,

(2) for each $\tau \in \mathfrak{h}^{*}, c_{x \tau}=0$ for all but finitely many $x \in X$. 
In this case, we define $\sum_{x \in X} f_{x}$ to be the element $\sum_{\tau \in h^{*}}\left(\sum_{x \in X} c_{x \tau}\right) e(\tau)$ of $\mathscr{E}$, and call it the sum of the $f_{x}$ 's.

Then we know the following.

Proposition 3.2.1 ([DGK, Proposition 3.4]). For a module $V$ in the category $\mathscr{O}$, there exists a unique set $\left\{a_{\mu}\right\}_{\mu \in \mathfrak{h}^{*}}$ of nonnegative integers such that the following equality holds in the algebra $\mathscr{E}$ :

$$
\operatorname{ch} V=\sum_{\mu \in \mathfrak{h}^{*}} a_{\mu} \operatorname{ch} L(\mu) .
$$

Definition 3.2.2 ([DGK, Definition 3.5(b)]). The above integer $a_{\mu}$ is called the multiplicity of $L(\mu)$ in $V$, and is denoted by $[V: L(\mu)]$.

Remark 3.2.3. It is clear that the above definition of the multiplicity $[V: L(\mu)]$ is identical with the one in [N], or [K, Chapter 9].

We note that for a module $V \in \mathscr{O}$ and $\mu \in \mathfrak{h}^{*},[V: L(\mu)] \neq 0$ if and only if $L(\mu)$ is an irreducible subquotient of $V$.

3.3. Some module-theoretic results on GKM algebras. Here we collect some results about the irreducible subquotients and embeddings of Verma modules over symmetrizable GKM algebras established in $[\mathrm{N}]$, which we shall use frequently later.

We choose and fix an element $\rho \in \mathfrak{h}^{*}$ such that $\left\langle\rho, \alpha_{i}^{\vee}\right\rangle=(1 / 2) \cdot a_{i i}$ for all $i \in I$, or equivalently, $\left(\rho \mid \alpha_{i}\right)=(1 / 2) \cdot\left(\alpha_{i} \mid \alpha_{i}\right)$ for all $i \in I$. From now on, we shall use the notation

$$
(w, \beta) \circ \Lambda:=w(\Lambda+\rho-\beta)-\rho
$$

for $(w, \beta) \in W \times \mathscr{A}$ and $\Lambda \in P_{+}$.

For the following results, the symmetrizability assumption on the GGCM $A$ is essential.

Theorem 3.3.1 ([N, Theorem 3.5]). Let $\Lambda \in P_{+},(w, \beta) \in W \times \mathscr{A}(\Lambda)$. Then any irreducible subquotient of the Verma module $V((w, \beta) \circ \Lambda)$ is isomorphic to $L\left(\left(w^{\prime}, \beta^{\prime}\right) \circ \Lambda\right)$ for some $\left(w^{\prime}, \beta^{\prime}\right) \in W \times \mathscr{A}(\Lambda)$ with $\left(w^{\prime}, \beta^{\prime}\right) \geqslant$ $(w, \beta)$. Conversely, for any $\left(w^{\prime}, \beta^{\prime}\right) \in W \times \mathscr{A}(\Lambda)$ with $\left(w^{\prime}, \beta^{\prime}\right) \geqslant(w, \beta)$, $L\left(\left(w^{\prime}, \beta^{\prime}\right) \circ \Lambda\right)$ is isomorphic to an irreducible subquotient of $V((w, \beta) \circ \Lambda)$.

Theorem 3.3.2 ([N, Theorem 3.7]). Fix $\Lambda \in P_{+}$. Let $\left(w_{1}, \beta_{1}\right),\left(w_{2}, \beta_{2}\right) \in$ $W \times \mathscr{A}(\Lambda)$. Then we have

$$
\operatorname{dim}_{\mathbb{C}} \operatorname{Hom}_{\mathfrak{g}(A)}\left(V\left(\left(w_{1}, \beta_{1}\right) \circ \Lambda\right), V\left(\left(w_{2}, \beta_{2}\right) \circ \Lambda\right)\right) \leq 1 .
$$

Note that any nonzero $\mathfrak{g}(A)$-module homomorphism between two Verma modules is injective. So we may write

$$
V\left(\left(w_{1}, \beta_{1}\right) \circ \Lambda\right) \subset V\left(\left(w_{2}, \beta_{2}\right) \circ \Lambda\right)
$$

when the equality holds in the above theorem.

Theorem 3.3.3 ([N, Theorem 3.9]). Let $\Lambda \in P_{+},\left(w_{1}, \beta_{1}\right),\left(w_{2}, \beta_{2}\right) \in W \times$ $\mathscr{A}(\Lambda)$. Then

$$
\begin{array}{ll} 
& V\left(\left(w_{1}, \beta_{1}\right) \circ \Lambda\right) \subset V\left(\left(w_{2}, \beta_{2}\right) \circ \Lambda\right) \\
\Longleftrightarrow \quad & \left(w_{1}, \beta_{1}\right) \geqslant\left(w_{2}, \beta_{2}\right) \\
\Longleftrightarrow \quad & {\left[V\left(\left(w_{2}, \beta_{2}\right) \circ \Lambda\right): L\left(\left(w_{1}, \beta_{1}\right) \circ \Lambda\right)\right] \neq 0 .}
\end{array}
$$




\section{Generalization OF JANTZEN'S CHARACTER SUM FORMULA}

In this section, we obtain a generalization to GKM algebras of Jantzen's character sum formula for a quotient of two Verma modules.

The original formula was proved by Jantzen [J] for finite-dimensional semisimple Lie algebras (over $\mathbb{C}$ ), and was generalized to the case of symmetrizable Kac-Moody algebras by Rocha-Caridi and Wallach [RW].

We now generalize this formula to the case of symmetrizable GKM algebras $\mathfrak{g}(A)$ associated to a GGCM $A=\left(a_{i j}\right)_{i, j \in I}$ satisfying the condition that $a_{i i} \neq 0$ $(i \in I)$. (This condition seems essential to us.) As mentioned earlier, under this condition, we may apply the proof (for the case of Kac-Moody algebras) by Rocha-Caridi and Wallach to the case of GKM algebras by making minor changes. So we shall give only a sketch of the proof, which, however, occupies the whole of $\S 4.3$.

4.1. Shapovalov form and its determinant. Here we recall the definition of the Shapovalov form on the universal enveloping algebra $U\left(\mathfrak{n}_{-}\right)$of the Lie subalgebra $\mathfrak{n}_{-}=\sum_{\alpha \in \Delta_{+}}^{\oplus} \mathfrak{g}_{-\alpha}$ of $\mathfrak{g}(A)$, and the result of Kac and Kazhdan [KK] about the determinant of the Shapovalov form restricted to each weight space $U\left(\mathfrak{n}_{-}\right)_{-\eta}$ for $\eta \in Q_{+}$.

Let $\mathfrak{g}(A)$ be the GKM algebra associated to a symmetrizable GGCM $A=$ $\left(a_{i j}\right)_{i, j \in I}$. Then the universal enveloping algebra $U(\mathfrak{g}(A))$ of $\mathfrak{g}(A)$ can be written as $U(\mathfrak{g}(A))=U\left(\mathfrak{n}_{-}\right) \otimes_{\mathbb{C}} U(\mathfrak{h}) \otimes_{\mathbb{C}} U\left(\mathfrak{n}_{+}\right)$, corresponding to the triangular decomposition: $\mathfrak{g}(A)=\mathfrak{n}_{-} \oplus \mathfrak{h} \oplus \mathfrak{n}_{+}$. In particular, we have the following decomposition as a vector space:

$$
U(\mathfrak{g}(A))=U(\mathfrak{h}) \oplus\left(\mathfrak{n}_{-} U(\mathfrak{g}(A))+U(\mathfrak{g}(A)) \mathfrak{n}_{+}\right) .
$$

We denote by $p: U(\mathfrak{g}(A)) \rightarrow U(\mathfrak{h})$ the Harish-Chandra projection of $U(\mathfrak{g}(A))$ onto $U(\mathfrak{h})$ parallel to the second summand of the above sum.

Then the Shapovalov form $F(\cdot, \cdot)$ with values in $U(\mathfrak{h})$ on $U(\mathfrak{g}(A))$ (see [S]) is defined by

$$
F(x, y):=p(\sigma(x) y) \quad(x, y \in U(\mathfrak{g}(A))),
$$

where $\sigma$ is the involutive anti-automorphism of $U(\mathfrak{g}(A))$ determined by $\sigma\left(e_{i}\right)$ $=f_{i}, \sigma\left(f_{i}\right)=e_{i} \quad(i \in I)$, and $\sigma(h)=h \quad(h \in \mathfrak{h})$.

Clearly, $F(\cdot, \cdot)$ is a symmetric bilinear $(U(\mathfrak{h})$-valued) form, such that

$$
F(z x, y)=F(x, \sigma(z) y) \quad(x, y, z \in U(\mathfrak{g}(A))) .
$$

In particular, we have

$$
F\left(U(\mathfrak{g}(A))_{\eta_{1}}, U(\mathfrak{g}(A))_{\eta_{2}}\right)=0 \quad \text { if } \quad \eta_{1} \neq \eta_{2} \in Q=\sum_{i \in I} \mathbb{Z} \alpha_{i},
$$

where $U(\mathfrak{g}(A))_{\eta}$ is the weight space of weight $\eta \in Q\left(\subset \mathfrak{h}^{*}\right)$ of $U(\mathfrak{g}(A))$ under the (extended) adjoint action of $\mathfrak{h}$.

Furthermore, on the Verma module $V(\lambda)\left(\lambda \in \mathfrak{h}^{*}\right)$, we define a symmetric bilinear $(\mathbb{C}$-valued $)$ form $(\cdot, \cdot)_{\lambda}($ see $[\mathrm{J}])$ by

$$
\left(g_{1} v_{\lambda}, g_{2} v_{\lambda}\right)_{\lambda}:=\lambda\left(F\left(g_{1}, g_{2}\right)\right) \quad\left(g_{1}, g_{2} \in U(\mathfrak{g}(A))\right),
$$

where $v_{\lambda}=1 \otimes 1 \in V(\lambda)$ is the canonical generator. Here $\lambda \in \mathfrak{h}^{*}$ is naturally extended to $U(\mathfrak{h})\left(\cong \mathbb{C}\left[\mathfrak{h}^{*}\right]\right)$ by: $\lambda\left(h_{1} h_{2} \cdots h_{k}\right):=\left\langle\lambda, h_{1}\right\rangle\left\langle\lambda, h_{2}\right\rangle \cdots\left\langle\lambda, h_{k}\right\rangle$ $\left(h_{1}, h_{2}, \ldots, h_{k} \in \mathfrak{h}\right)$. 
One can easily check that the form $(\cdot, \cdot)_{\lambda}$ is well-defined, and satisfies the following properties:

(1) $\left(g v_{1}, v_{2}\right)_{\lambda}=\left(v_{1}, \sigma(g) v_{2}\right)_{\lambda} \quad\left(g \in U(\mathfrak{g}(A)), v_{1}, v_{2} \in V(\lambda)\right)$;

(2) $\left(V(\lambda)_{\tau_{1}}, V(\lambda)_{\tau_{2}}\right)_{\lambda}=0 \quad\left(\tau_{1} \neq \tau_{2} \in \mathfrak{h}^{*}\right)$;

(3) the radical of $(\cdot, \cdot)_{\lambda}$ is a unique maximal proper $\mathfrak{g}(A)$-submodule of $V(\lambda)$.

This form $(\cdot, \cdot)_{\lambda}$ on the Verma module $V(\lambda)$ is also called a Shapovalov form.

For $\eta \in Q_{+}$, we denote by $F_{\eta}$ the restriction of the Shapovalov form $F$ to the weight space $U\left(\mathfrak{n}_{-}\right)_{-\eta}:=U\left(\mathfrak{n}_{-}\right) \cap U(\mathfrak{g}(A))_{-\eta}$. Note that $\operatorname{dim}_{\mathbb{C}} U\left(\mathfrak{n}_{-}\right)_{-\eta}=$ $K(\eta)<+\infty$, where $K(\eta) \quad\left(\eta \in Q_{+}\right)$is the (generalized) Kostant partition function (see [K, Chapter 10]).

We know the following theorem, due to Kac and Kazhdan [KK].

Theorem 4.1.1 ([KK, Theorem 1]). We keep the above notation. Then, up to a nonzero constant factor (depending on the basis of $U\left(\mathfrak{n}_{-}\right)_{-\eta}$ ), we have

$$
\operatorname{det} F_{\eta}=\prod_{\beta \in \Delta_{+}} \prod_{j=1}^{\infty}\left(2\left(\nu^{-1}(\beta)+(\rho \mid \beta)\right)-j(\beta \mid \beta)\right)^{K(\eta-j \beta)},
$$

where the roots $\beta \in \Delta_{+}$are taken with their multiplicities.

Remark 4.1.2. In [KK], the above theorem is proved in the more general setting of contragredient Lie algebras.

4.2. Jantzen's character sum formula for a single Verma module. Here we review the theory of Jantzen's character sum formula for a single Verma module (cf. [J]). This was generalized to the case of contragredient Lie algebras by Kac and Kazhdan [KK], by using Theorem 4.1.1 above. However, we prefer to use a slightly different derivation of the filtration of a Verma module given by RochaCaridi and Wallach, which may be thought of as a special case of the filtration given in $\S 4.3$ (see [RW] and [W]).

Let $\mathfrak{g}(A)$ be a symmetrizable GKM algebra. Recall that the universal enveloping algebra $U\left(\mathfrak{n}_{-}\right)$of $\mathfrak{n}_{-}$is isomorphic to Verma modules $V(\lambda) \quad\left(\lambda \in \mathfrak{h}^{*}\right)$ as a vector space via the map $U\left(\mathfrak{n}_{-}\right) \rightarrow V(\lambda)$ given by $y \mapsto y \otimes 1=y v_{\lambda}$, where $v_{\lambda}=1 \otimes 1 \in V(\lambda)$ is the canonical generator. Then, via the above map, we can regard $U\left(\mathfrak{n}_{-}\right)$as a $\mathfrak{g}(A)$-module with an action of $\mathfrak{g}(A)$ denoted by $\pi_{\lambda}$. (Here we remark that for $y \in U\left(\mathfrak{n}_{-}\right)_{-\eta}\left(\eta \in Q_{+}\right)$and $h \in \mathfrak{h}$, we have $\pi_{\lambda}(h) y=$ $\langle\lambda-\eta, h\rangle y$.) Moreover, again via the above map, the Shapovalov form $(\cdot, \cdot)_{\lambda}$ can also be regarded as a bilinear form on $U\left(\mathfrak{n}_{-}\right)$(depending on $\left.\lambda \in \mathfrak{h}^{*}\right)$, such that $\left(\pi_{\lambda}(g) x, y\right)_{\lambda}=\left(x, \pi_{\lambda}(\sigma(g)) y\right)_{\lambda} \quad\left(g \in U(\mathfrak{g}(A)), x, y \in U\left(\mathfrak{n}_{-}\right)\right)$.

Now we look upon $\mathbb{C}[t] \otimes_{\mathbb{C}} U\left(\mathfrak{n}_{-}\right)=\sum_{\eta \in Q_{+}}^{\oplus} \mathbb{C}[t] \otimes_{\mathbb{C}} U\left(\mathfrak{n}_{-}\right)_{-\eta}$ as the space of all polynomials in one variable $t$ with values in $U\left(\mathfrak{n}_{-}\right)$. We fix an element $\zeta$ of $\mathfrak{h}^{*}$ such that $(\zeta \mid \eta) \neq 0$ for any $\eta \in Q_{+} \backslash\{0\}$.

Then we have the following.

Theorem 4.2.1 (see [J], [KK], [W]). We keep the above notation. Set $V(\lambda)_{i}:=$ $\left\{f(0) v_{\lambda} \in V(\lambda) \mid f \in \mathbb{C}[t] \otimes_{\mathbb{C}} U\left(\mathfrak{n}_{-}\right),(f(t), x)_{\lambda+t \zeta} \in t^{i} \mathbb{C}[t]\right.$ for all $\left.y \in U\left(\mathfrak{n}_{-}\right)\right\}$ for $i \geq 0$. Then the filtration

$$
V(\lambda)=V(\lambda)_{0} \supset V(\lambda)_{1} \supset V(\lambda)_{2} \supset \cdots
$$


defines a $\mathfrak{g}(A)$-module filtration of $V(\lambda)$ such that

(1) for any $\eta \in Q_{+}, V(\lambda)_{\lambda-\eta} \cap V(\lambda)_{i}=0$ for some $i$,

(2) $V(\lambda) / V(\lambda)_{1} \cong L(\lambda)$ as a $\mathfrak{g}(A)$-module,

(3) the following equality holds in the algebra $\mathscr{E}$ :

$$
\sum_{i \geq 1} \operatorname{ch} V(\lambda)_{i}=\sum_{\beta \in \Delta_{+}} \sum_{\substack{l \in \mathbb{Z}_{\geq 1} \\ 2(\lambda+\rho \mid \beta)=l(\beta \mid \beta)}} \operatorname{ch} V(\lambda-l \beta),
$$

where the roots $\beta \in \Delta_{+}$are taken with their multiplicities.

The above filtration $V(\lambda)=V(\lambda)_{0} \supset V(\lambda)_{1} \supset V(\lambda)_{2} \supset \cdots \quad$ is called Jantzen's filtration of $V(\lambda)$, and the assertion (3) is called Jantzen's character sum formula.

4.3. Generalization of Jantzen's character sum formula for a quotient of two Verma modules. The aim of this subsection is to sketch the proof of a generalization (to GKM algebras) of Jantzen's character sum formula for a quotient of two Verma modules ( $=$ the first assertion of Theorem II in the Introduction). The proof is based on a number of lemmas below.

From now till the end of this paper, we assume that the GGCM $A=\left(a_{i j}\right)_{i, j \in I}$ is symmetrizable, and satisfies the condition that $a_{i i} \neq 0$ for $i \in I$. This assumption is crucial for the proofs of some lemmas, and of course, for the main result (Theorem 4.3.12) of this subsection.

First we recall the following useful lemma.

Lemma 4.3.1 (see [N, Lemma 3.3 and Remark 3.4]). Let $\mu \in P_{+}, w \in W$, and $\gamma \in \Delta_{+}$. Then the following are equivalent:

(1) $2(w(\mu+\rho) \mid \gamma)=m(\gamma \mid \gamma)$ for some $m \in \mathbb{Z}_{\geq 1}$;

(2) we have either of the following two cases:

(a) $\gamma \in \Delta^{r e}$ and $\ell\left(r_{\gamma} w\right)>\ell(w)$,

(b) $w^{-1}(\gamma) \in \Pi^{i m}$ and $\left(w^{-1}(\gamma) \mid \mu\right)=0$.

Moreover, in case (a), we have $r_{\gamma} w \geqslant w$ and $m=\left\langle w(\mu+\rho), \gamma^{\vee}\right\rangle$. In case (b), we have $m=1$.

Now we introduce some notation, following [RW]. We set

$$
\Gamma:=\bigcup_{w \in W}(w, 0) \circ P_{+},
$$

and then, for each $i \in I^{r e}$,

$$
\Gamma_{i}^{+}:=\left\{\lambda \in \Gamma \mid\left\langle\lambda+\rho, \alpha_{i}^{\vee}\right\rangle>0\right\} \text { and } \Gamma_{i}^{-}:=\left\{\lambda \in \Gamma \mid\left\langle\lambda+\rho, \alpha_{i}^{\vee}\right\rangle<0\right\} .
$$

In addition, for $\alpha \in \Delta$ and $k \in \mathbb{Z}_{\geq 1}$, we put

$$
\mathfrak{h}_{\alpha, k}^{*}:=\left\{\lambda \in \mathfrak{h}^{*} \mid 2(\lambda+\rho \mid \alpha)=k(\alpha \mid \alpha)\right\} .
$$

If $\alpha \in W \cdot \Pi^{i m}\left(\subset \Delta_{+} \cap \Delta^{i m}\right)$, we put

$$
m(\alpha):=\min \left\{\ell(w) \mid \alpha=w\left(\alpha_{j}\right)\left(w \in W, j \in I^{i m}\right)\right\} .
$$

Here we note that $w_{1}\left(\alpha_{j_{1}}\right)=w_{2}\left(\alpha_{j_{2}}\right) \quad\left(w_{1}, w_{2} \in W, j_{1}, j_{2} \in I^{i m}\right)$ implies $\alpha_{j_{1}}=\alpha_{j_{2}}$, since $\left\langle\alpha_{j_{1}}, \alpha_{i}^{\vee}\right\rangle,\left\langle\alpha_{j_{2}}, \alpha_{i}^{\vee}\right\rangle \leq 0\left(i \in I^{r e}\right)$ (cf. the proof of [K, Proposition 3.12]). We also note that, for $\alpha=w\left(\alpha_{j}\right) \quad\left(w \in W, \alpha_{j} \in \Pi^{i m}\right), k \alpha \in \Delta$ 
if and only if $k= \pm 1$, and $\operatorname{dim}_{\mathbb{C}} \mathfrak{g}_{\alpha}=\operatorname{dim}_{\mathbb{C}} \mathfrak{g}_{\alpha_{j}}=1$, since the root system $\Delta$ is invariant with their multiplicities under the action of the Weyl group $W$.

Remark 4.3.2. Let $\alpha \in W \cdot \Pi^{i m}$, and let $j \in I^{i m}$ and $w \in W$ be such that $\alpha=w\left(\alpha_{j}\right)$ with $\ell(w)=m(\alpha)$. Then $\Lambda \in P_{+} \cap \mathfrak{h}_{\alpha_{j}, k}^{*}$ implies that

$$
k\left(\alpha_{j} \mid \alpha_{j}\right)=2\left(\Lambda+\rho \mid \alpha_{j}\right)=2\left(\Lambda \mid \alpha_{j}\right)+\left(\alpha_{j} \mid \alpha_{j}\right),
$$

so that

$$
0 \leq 2\left(\Lambda \mid \alpha_{j}\right)=(k-1) \cdot\left(\alpha_{j} \mid \alpha_{j}\right) \leq 0 .
$$

Thus, $P_{+} \cap \mathfrak{h}_{\alpha_{j}, k}^{*}$ is Zariski-dense in $\mathfrak{h}_{\alpha_{j}, k}^{*}$ if and only if $k=1$, since $\left(\alpha_{j} \mid \alpha_{j}\right)<$ 0 .

The proof of the following lemma is essentially the same as that of [RW, Lemma 3.2], except that we use Remark 4.3.2 above.

Lemma 4.3.3 (cf. [RW, Lemma 3.2]). If $\alpha \in W \cdot \Pi^{\text {im }}$ is not an imaginary simple root, then there exists some $i \in I^{r e}$ such that

(1) $m\left(r_{i}(\alpha)\right)<m(\alpha)$,

(2) $\Gamma_{i}^{-} \cap \mathfrak{h}_{\alpha, 1}^{*}$ is Zariski-dense in $\mathfrak{h}_{\alpha, 1}^{*}$.

We now record (for later use) the following formula, which holds for an arbitrary $i \in I$ :

$$
\left[e_{i}, f_{i}^{k}\right]=k f_{i}^{k-1}\left(\alpha_{i}^{\vee}-(k-1) \cdot a_{i i} / 2\right) \quad(i \in I) .
$$

Theorem 4.3.4 (cf. [S, Lemma 1] and [RW, Theorem 3.3]). Let $\alpha=\sum_{i \in I} m_{i} \alpha_{i} \in$ $W \cdot \Pi^{i m}$. Then there exists $\theta_{\alpha, 1} \in U\left(\mathfrak{n}_{-} \oplus \mathfrak{h}\right)_{-\alpha}=U\left(\mathfrak{n}_{-} \oplus \mathfrak{h}\right) \cap U(\mathfrak{g}(A))_{-\alpha}$ satisfying:

(1) $\left[e_{i}, \theta_{\alpha, 1}\right] \in U(\mathfrak{g}(A))\left(\nu^{-1}(\alpha)+(\rho \mid \alpha)-(1 / 2) \cdot(\alpha \mid \alpha)\right)+U(\mathfrak{g}(A)) \mathfrak{n}_{+}$for $i \in I$

(2) $\theta_{\alpha, 1}=f_{1}^{m_{1}} f_{2}^{m_{2}} \cdots f_{n}^{m_{n}}+\sum_{l} a_{l} b_{l}$, where $a_{l} \in U_{p}\left(\mathfrak{n}_{-}\right), b_{l} \in U(\mathfrak{h})$ with $p<\sum_{i \in I} m_{i}$. Here $\left\{U_{p}\left(\mathfrak{n}_{-}\right)\right\}_{p \geq 0}$ is the canonical filtration of $U\left(\mathfrak{n}_{-}\right)$.

Sketch of proof. The proof is by induction on $m(\alpha)$. If $m(\alpha)=0$, then obviously $\alpha=\alpha_{j}$ for some $j \in I^{i m}$. In this case, we can clearly take $f_{j}$ as $\theta_{\alpha_{j}, 1}$.

Suppose that $m(\alpha) \geq 1$. Then, by Lemma 4.3.3, there exists some $i \in I^{r e}$ such that $m\left(r_{i}(\alpha)\right)<m(\alpha)$ and such that $\Gamma_{i}^{-} \cap \mathfrak{h}_{\alpha, 1}^{*}$ is Zariski-dense in $\mathfrak{h}_{\alpha, 1}^{*}$.

Now, for each $\lambda \in \Gamma_{i}^{-} \cap \mathfrak{h}_{\alpha, 1}^{*}$, we can construct an element $\theta_{\alpha, 1}(\lambda) \in U\left(\mathfrak{n}_{-}\right)_{-\alpha}$

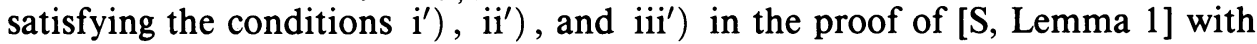
$B$ replaced by $\Gamma_{i}^{-} \cap \mathfrak{h}_{\alpha, 1}^{*}$. (and $L_{\alpha, 1}$ by $\mathfrak{h}_{\alpha, 1}^{*}$ ) as follows.

We write $\lambda=(w, 0) \circ \Lambda$ for $w \in W$ and $\Lambda \in P_{+}$. Put $\psi:=\left(r_{i}, 0\right) \circ \lambda=$ $\left(r_{i} w, 0\right) \circ \Lambda$. Since $\lambda \in \mathfrak{h}_{\alpha, 1}^{*}$, i.e.,

$$
(\alpha \mid \alpha)=2(\lambda+\rho \mid \alpha)=2(w(\Lambda+\rho) \mid \alpha),
$$

we get $\alpha=w\left(\alpha_{k}\right)$ for some $\alpha_{k} \in \Pi^{i m}$ with $\left(\Lambda \mid \alpha_{k}\right)=0$, by Lemma 4.3.1. Furthermore, since $\lambda \in \Gamma_{i}^{-}$, i.e.,

$$
0>\left\langle w(\Lambda+\rho), \alpha_{i}^{\vee}\right\rangle=2\left(w(\Lambda+\rho) \mid \alpha_{i}\right) /\left(\alpha_{i} \mid \alpha_{i}\right),
$$


we get $w \geqslant r_{i} w$ again by Lemma 4.3.1. So we have

$$
\begin{aligned}
\lambda-\alpha & =w(\Lambda+\rho)-\rho-w\left(\alpha_{k}\right) \\
& =w\left(\Lambda+\rho-\alpha_{k}\right)-\rho \\
& =\left(w, \alpha_{k}\right) \circ \Lambda,
\end{aligned}
$$

and

$$
\begin{aligned}
\psi-r_{i}(\alpha) & =\left(r_{i} w\right)(\Lambda+\rho)-\rho-r_{i} w\left(\alpha_{k}\right) \\
& =\left(r_{i} w\right)\left(\Lambda+\rho-\alpha_{k}\right)-\rho \\
& =\left(r_{i} w, \alpha_{k}\right) \circ \Lambda
\end{aligned}
$$

with $w \geqslant r_{i} w$.

Therefore, by Theorems 3.3.2 and 3.3.3, we have two series of inclusions:

$$
\begin{gathered}
V(\lambda-\alpha) \hookrightarrow V(\lambda) \hookrightarrow V(\psi), \\
V(\lambda-\alpha) \hookrightarrow V\left(\psi-r_{i}(\alpha)\right) \hookrightarrow V(\psi),
\end{gathered}
$$

(where the corresponding composite maps $V(\lambda-\alpha) \hookrightarrow V(\psi)$ are identical. From this, using the induction hypothesis (since $m\left(r_{i}(\alpha)\right)<m(\alpha)$ ), we obtain the desired element $\theta_{\alpha, 1}(\lambda) \in U\left(\mathfrak{n}_{-}\right)_{-\alpha}$, as in the proof of [S, Lemma 1] (we take $\varepsilon=\alpha_{i}$ ).

Now the rest of the argument in the proof of [S, Lemma 1] goes through without any serious change.

Let $\chi: \mathfrak{n}_{-} \rightarrow \mathbb{C}$,be the linear map defined by $\chi\left(f_{i}\right)=1 \quad(i \in I)$, and $\chi\left(\left[\mathfrak{n}_{-}, \mathfrak{n}_{-}\right]\right)=0$. We extend $\chi$ to the algebra homomorphism from $U\left(\mathfrak{n}_{-}\right)$to $\mathbb{C}$, which is again denoted by $\chi$.

For $\lambda \in \mathfrak{h}^{*}$, let $(\cdot, \cdot)_{\lambda}$ be the Shapovalov form on the Verma module $V(\lambda)$, which we also regard as a bilinear form on the universal enveloping algebra $U\left(\mathfrak{n}_{-}\right)$, as in $\S 4.2$.

Let $\mathscr{D}:=\left\{\lambda \in \mathfrak{h}^{*} \mid V(\lambda)\right.$ is irreducible $\}$. Then, by results of Kac and Kazhdan $[\mathrm{KK}]$, we have

$$
\mathscr{D}=\left\{\lambda \in \mathfrak{h}^{*} \mid 2(\lambda+\rho \mid \alpha) \neq k(\alpha \mid \alpha) \text { for any } \alpha \in \Delta_{+} \text {and } k \in \mathbb{Z}_{\geq 1}\right\} .
$$

Before proceeding further, we prepare the following notation: for the Verma module $V(\lambda)=\sum_{\tau \in \mathfrak{h}^{*}}^{\oplus} V(\lambda)_{\tau}$, we put $V(\lambda)_{\left[\mathfrak{n}_{-}\right]}:=\prod_{\tau \in \mathfrak{h}^{*}} V(\lambda)_{\tau}$ (direct product of weight spaces). Then the $\mathfrak{h}$-module structure on the vector space $V(\lambda)_{\left[\mathfrak{n}_{-}\right]}$ can be extended in the natural way to a $\mathfrak{g}(A)$-module structure.

The proofs of the next Lemmas 4.3 .5 and 4.3.6 are essentially the same as those of [GW, Lemma 4.1] and [GW, Corollary 4.3], respectively. However, we give some detail for the convenience of the reader, since our setting differs considerably from that of [GW].

Lemma 4.3.5 (cf. [GW, Lemma 4.1] and [RW, Lemma 3.5]). For each $\mu \in Q_{+}$, there exists a rational function $\lambda \mapsto \bar{n}_{-\mu}(\lambda)$ from $\mathfrak{h}^{*}$ into $U\left(\mathfrak{n}_{-}\right)_{-\mu}$ with the following properties:

(1) $\bar{n}_{0}(\lambda) \equiv 1$

(2) if $\lambda$ is in $\mathscr{D}$, then $\bar{n}_{-\mu}$ is defined at $\lambda$, and we have $\left(y, \bar{n}_{-\mu}(\lambda)\right)_{\lambda}=\chi(y)$ for $y \in U\left(\mathfrak{n}_{-}\right)_{-\mu}$; 
(3) for $i \in I$, there is a rational function $\lambda \mapsto \bar{n}_{-\mu, i}^{\prime}(\lambda)$ from $\mathfrak{h}^{*}$ into $U\left(\mathfrak{n}_{-}\right)_{-\mu+\alpha_{i}}$, whose singularity set is contained in that of $\bar{n}_{-\mu}$, such that for $\lambda \in \mathscr{D}$,

$$
\left[e_{i}, \bar{n}_{-\mu}(\lambda)\right]=\bar{n}_{-\mu+\alpha_{i}}(\lambda)+\bar{n}_{-\mu, i}^{\prime}(\lambda)\left(\alpha_{i}^{\vee}-\left\langle\lambda, \alpha_{i}^{\vee}\right\rangle\right) .
$$

Sketch of proof. If $V(\lambda)$ is irreducible, then the Shapovalov form $(\cdot, \cdot)_{\lambda}$ on $V(\lambda)$ is clearly nondegenerate. Moreover, we can naturally extend $(\cdot, \cdot)_{\lambda}$ to a pairing (again denoted by $\left.(\cdot, \cdot)_{\lambda}\right)$ of $U\left(\mathfrak{n}_{-}\right)$with $V(\lambda)_{\left[\mathfrak{n}_{-}\right]}$, such that $(y X, v)_{\lambda}$ $=(X, \sigma(y) v)_{\lambda} \quad\left(X, y \in U\left(\mathfrak{n}_{-}\right), v \in V(\lambda)_{\left[\mathfrak{n}_{-}\right]}\right)$.

In this case, the map $v \mapsto(\cdot, v)_{\lambda}$ defines a linear isomorphism $\phi: V(\lambda)_{\left[\mathfrak{n}_{-}\right]} \rightarrow$ $\operatorname{Hom}_{\mathbb{C}}\left(U\left(\mathfrak{n}_{-}\right), \mathbb{C}\right)$. Since $\chi$ is an element of $\operatorname{Hom}_{\mathbb{C}}\left(U\left(\mathfrak{n}_{-}\right), \mathbb{C}\right)$, there exists a unique element $v_{0} \in V(\lambda)_{\left[n_{-}\right]}$such that $\phi\left(v_{0}\right)=\chi$, i.e., $\left(y, v_{0}\right)_{\lambda}=\chi(y)$ $\left(y \in U\left(\mathfrak{n}_{-}\right)\right)$. Here we have, for $x \in U\left(\mathfrak{n}_{+}\right), y \in U\left(\mathfrak{n}_{-}\right)$,

$$
\begin{aligned}
\left(y, x v_{0}\right)_{\lambda} & =\left(\sigma(x) y, v_{0}\right)_{\lambda}=\chi(\sigma(x) y)=\chi(\sigma(x)) \chi(y) \\
& =\chi(\sigma(x))\left(y, v_{0}\right)_{\lambda}=\left(y, \chi(\sigma(x)) v_{0}\right)_{\lambda},
\end{aligned}
$$

so that $x v_{0}=\chi(\sigma(x)) v_{0}$.

Because $v_{0}$ is an element of $V(\lambda)_{\left[\mathfrak{n}_{-}\right]}$, we can write

$$
v_{0}=\sum_{\mu \in Q_{+}} \bar{n}_{-\mu}(\lambda) v_{\lambda},
$$

where $v_{\lambda}$ is the canonical generator of $V(\lambda)$, and for $\mu \in Q_{+}, \bar{n}_{-\mu}(\lambda) \in$ $U\left(\mathfrak{n}_{-}\right)_{-\mu}$ is uniquely determined by the property

$$
\left(y, \bar{n}_{-\mu}(\lambda)\right)_{\lambda}=\chi(y) \quad\left(y \in U\left(\mathfrak{n}_{-}\right)_{-\mu}\right) .
$$

To show that $\bar{n}_{-\mu}(\lambda)$ depends rationally on $\lambda$, we define a positive definite inner product $(\cdot, \cdot)$ on $U\left(\mathfrak{n}_{-}\right)$in a natural way, using a basis of $U\left(\mathfrak{n}_{-}\right)$constructed from root vectors by the Poincare-Birkhoff-Witt theorem. Then, for $\mu=\sum_{i \in I} m_{i} \alpha_{i} \in Q_{+}$, there exists a polynomial map $\lambda \mapsto \tau_{\mu}(\lambda)$ from $\mathfrak{h}^{*}$ into $\operatorname{End}_{\mathbb{C}}\left(U\left(\mathfrak{n}_{-}\right)_{-\mu}\right)$ such that

$$
\left(y, y^{\prime}\right)_{\lambda}=\left(y, \tau_{\mu}(\lambda) y^{\prime}\right) \quad\left(y, y^{\prime} \in U\left(\mathfrak{n}_{-}\right)_{-\mu}\right) .
$$

If $\lambda \in \mathscr{D}$, i.e., $V(\lambda)$ is irreducible, then $(\cdot, \cdot)_{\lambda}$ is nondegenerate on $U\left(\mathfrak{n}_{-}\right)_{-\mu}$, so $\operatorname{det} \tau_{\mu}(\lambda) \neq 0$ for all $\mu \in Q_{+}$. Furthermore, in this case, we see from the above argument that $\bar{n}_{-\mu}(\lambda)=\tau_{\mu}(\lambda)^{-1} f_{1}^{m_{1}} f_{2}^{m_{2}} \cdots f_{n}^{m_{n}}$.

Finally, the assertion (3) follows from the fact that $x v_{0}=\chi(\sigma(x)) v_{0} \quad(x \in$ $\left.\mathfrak{n}_{+}\right)$by the same argument as in the proof of [GW, Lemma 4.1]. Thus, we have completed the proof of the lemma.

In order to derive a formula for $\bar{n}_{-\mu}(\lambda)$, we prepare some more notation. For $\mu=\sum_{i \in I} m_{i} \alpha_{i} \in Q_{+} \backslash\{0\}$, let $\operatorname{ht}(\mu):=\sum_{i \in I} m_{i}$ and set $m:=\operatorname{ht}(\mu)$. Let $\mathbb{J}(\mu):=\left\{\left(i_{1}, \ldots, i_{m}\right) \mid 1 \leq i_{j} \leq n\right.$ and $\left.\sum_{j=1}^{m} \alpha_{i_{j}}=\mu\right\}$. For $J \in \mathbb{J}(\mu)$ and $k$ $(1 \leq k \leq m)$, we set $\mu_{J_{k}}:=\sum_{j=k}^{m} \alpha_{i_{j}}=\mu-\sum_{j=1}^{k-1} \alpha_{i_{j}}, f_{J}:=f_{i_{1}} \cdots f_{i_{m}}$, and $e_{J}:=e_{i_{m}} \cdots e_{i_{1}}$. Define, for $\lambda \in \mathfrak{h}^{*}, \mu \in Q_{+} \backslash\{0\}$, and $J \in \mathbb{J}(\mu)$,

$$
P_{J}(\lambda, \mu):=\prod_{k=1}^{m}\left(2(\lambda+\rho)-\mu_{J_{k}} \mid \mu_{J_{k}}\right) \text {. }
$$


In addition, we set

$$
\mathscr{D}^{\prime}:=\left\{\lambda \in \mathfrak{h}^{*} \mid L_{\mu}(\lambda) \neq 0 \text { for all } \mu \in Q_{+} \backslash\{0\}\right\},
$$

where $L_{\mu}(\lambda):=(2(\lambda+\rho)-\mu \mid \mu)$. (Note that $\mathscr{D}^{\prime} \subset \mathscr{D}$.)

Then we have the following.

Lemma 4.3.6 (cf. [GW, Corollary 4.3], [RW, Lemma 3.6]). Let $\mu=\sum_{i \in I} m_{i} \alpha_{i}$ $\in Q_{+} \backslash\{0\}$, and suppose that $\lambda \in \mathscr{D}^{\prime}$. Then we have

$$
\bar{n}_{-\mu}(\lambda)=2^{\mathrm{ht}(\mu)} \cdot \sum_{J \in \mathbb{J}(\mu)} P_{J}(\lambda, \mu)^{-1} f_{J}
$$

Sketch of proof. Since $\lambda \in \mathscr{D}^{\prime} \subset \mathscr{D}$, the Verma module $V(\lambda)$ is irreducible. In the proof of Lemma 4.3.5, we have shown that the element $v_{0}=\sum_{\mu \in Q_{+}} \bar{n}_{-\mu}(\lambda) v_{\lambda}$ $\in V(\lambda)_{\left[\mathfrak{n}_{-}\right]}$satisfies $x v_{0}=\chi(\sigma(x)) v_{0}$ for $x \in \mathfrak{n}_{+}$. Therefore, it is obvious that the vectors $\bar{n}_{-\mu}(\lambda) v_{\lambda} \quad\left(\mu \in Q_{+}\right)$are annihilated by the derived subalgebra $\left[\mathfrak{n}_{+}, \mathfrak{n}_{+}\right]$of $\mathfrak{n}_{+}$.

Here we recall from [K, Chapter 2] that the (generalized) Casimir operator $\Omega$ is given by

$$
\Omega=2 \nu^{-1}(\rho)+\sum_{i} u^{i} u_{i}+\sum_{\alpha \in \Delta_{+}} \sum_{i} e_{-\alpha}^{(i)} e_{\alpha}^{(i)},
$$

where $\left\{u_{i}\right\}_{i}$ and $\left\{u^{i}\right\}_{i}$ are dual bases of $\mathfrak{h}$ with respect to $(\cdot \mid \cdot)$, and for $\alpha \in \Delta_{+}$, $\left\{e_{\alpha}^{(i)}\right\}_{i}$ and $\left\{e_{-\alpha}^{(i)}\right\}_{i}$ are bases of $\mathfrak{g}_{\alpha}$ and $\mathfrak{g}_{-\alpha}$, respectively, such that $\left(e_{\alpha}^{(i)} \mid e_{-\alpha}^{(j)}\right)=$ $\delta_{i j}$.

Let $v \in V(\lambda)_{\lambda-\mu}$ be an $\left[\mathfrak{n}_{+}, \mathfrak{n}_{+}\right]$-invariant for $\mu \in Q_{+}$. Then, clearly we have $e_{\alpha}^{(i)} v=0$ for $\alpha \in \Delta_{+} \backslash \Pi$. Therefore, from the fact that the Casimir operator $\Omega$ acts on $V(\lambda)$ by the scalar $(\lambda+\rho \mid \lambda+\rho)-(\rho \mid \rho)$, we can easily deduce that $L_{\mu}(\lambda) v=\sum_{i \in I} 2 f_{i} e_{i} v$. Then, by the same argument as in the proof of [GW, Lemma 4.2], we obtain

$$
v=c(\mu) \cdot \sum_{J \in \mathbb{J}(\mu)} P_{J}(\lambda, \mu)^{-1} f_{J} v_{\lambda}
$$

for some constant $c(\mu) \in \mathbb{C}$. Furthermore, the constant $c(\mu)$ is determined by

$$
2^{\mathrm{ht}(\mu)} e_{1}^{m_{1}} \cdots e_{n}^{m_{n}} v=c(\mu) v_{\lambda}
$$

Now we can apply this argument to the vector $\bar{n}_{-\mu}(\lambda) v_{\lambda}$, since the vector $\bar{n}_{-\mu}(\lambda) v_{\lambda} \in V(\lambda)_{\lambda-\mu}$ is an $\left[\mathfrak{n}_{+}, \mathfrak{n}_{+}\right]$-invariant, as remaked above. In this case, we can show that $c(\mu)=2^{\mathrm{ht}(\mu)}$, arguing as in the proof of [GW, Corollary 4.3]. This completes the proof of the lemma.

For notational simplicity, we put

$$
\mathbb{Z}_{\geq 1} \cdot \Delta_{+}:=\left\{\gamma \in Q_{+} \mid \gamma=j \alpha \text { for some } j \in \mathbb{Z}_{\geq 1} \text { and } \alpha \in \Delta_{+}\right\} \text {. }
$$

Set for $\mu \in Q_{+} \backslash\{0\}$,

$$
R_{\mu}:=\prod_{\substack{\gamma \in \mathbb{Z}_{\geq 1} \cdot \Delta_{+} \\ \gamma \leq \mu}} L_{\gamma}
$$


Lemma 4.3.7 (cf. [GW, Lemma 4.4] and [RW, Lemma 3.7]). Let $\mu \in Q_{+} \backslash\{0\}$. Then the function $S_{-\mu}(\lambda):=R_{\mu}(\lambda) \bar{n}_{-\mu}(\lambda)$ extends to a polynomial map from $\mathfrak{h}^{*}$ into $U\left(\mathfrak{n}_{-}\right)_{-\mu}$.

Sketch of proof. We put for $\mu \in Q_{+} \backslash\{0\}$,

$$
P_{\mu}:=\left\{\lambda \in \mathfrak{h}^{*} \mid L_{\mu}(\lambda)=0\right\} .
$$

First, we show that, for $0 \neq \mu \in Q_{+} \backslash \mathbb{Z}_{\geq 1} \cdot \Delta_{+}$and $\gamma \in \mathbb{Z}_{\geq 1} \cdot \Delta_{+}$, we have $P_{\mu} \neq P_{\gamma}$. Here it is obvious that for $\mu \in Q_{+} \backslash\{0\}$,

$$
P_{\mu}=\operatorname{Ker}(\mu \mid \cdot)+(1 / 2) \mu-\rho,
$$

where $\operatorname{Ker}(\mu \mid \cdot):=\left\{\lambda \in \mathfrak{h}^{*} \mid(\mu \mid \lambda)=0\right\}$.

Suppose that $P_{\mu}=P_{j \alpha}$ for some $0 \neq \mu \in Q_{+} \backslash \mathbb{Z}_{\geq 1} \cdot \Delta_{+}$and $j \in \mathbb{Z}_{\geq 1}, \alpha \in \Delta_{+}$. Then we have that $\operatorname{Ker}(\mu \mid \cdot)=\operatorname{Ker}(j \alpha \mid \cdot)=\operatorname{Ker}(\alpha \mid \cdot)$ and that $\mu-j \alpha \in \operatorname{Ker}(\alpha \mid \cdot)$. Because $(\cdot \mid \cdot)$ is nondegenerate on $\mathfrak{h}^{*}, \operatorname{Ker}(\mu \mid \cdot)=\operatorname{Ker}(\alpha \mid \cdot)$ implies $\mu=q \alpha$ for some positive rational number $q \in \mathbb{Q}$.

In the case where $(\alpha \mid \alpha) \neq 0$, we have

$$
0=(\alpha \mid \mu-j \alpha)=(q-j)(\alpha \mid \alpha),
$$

and hence $q=j$. This is a contradiction, since $\mu \notin \mathbb{Z}_{\geq 1} \cdot \Delta_{+}$.

In the case where $(\alpha \mid \alpha)=0$, we have

$$
\alpha \in \Delta_{+} \cap \Delta^{i m}=\bigcup_{w \in W} w(K),
$$

where $K$ is as in $\S 1.4$. So we have $\alpha=w(\tau)$ for some $w \in W, \tau \in K$. Here note that $\mu=q \alpha=w(q \tau) \in Q_{+} \backslash\{0\}$, and that $q$ is a positive rational number. Then it is obvious that $q \tau \in Q_{+} \backslash\{0\},\left\langle q \tau, \alpha_{i}^{\vee}\right\rangle \geq 0\left(i \in I^{r e}\right)$, and $\operatorname{supp}(q \tau)$ is connected. In addition, we have $q \tau \notin \bigcup_{j \geq 2} j \cdot \Pi^{i m}$, since we have assumed that $a_{i i} \neq 0 \quad(i \in I)$. Thus, we get $q \tau \in K$, so that $\mu$ itself is a positive imaginary root. This is a contradiction, since $\mu \notin \mathbb{Z}_{\geq 1} \cdot \Delta_{+}$.

From the above remark, we see immediately that, for $0 \neq \mu \in Q_{+} \backslash \mathbb{Z}_{\geq 1} \cdot \Delta_{+}$ and $\gamma \in \mathbb{Z}_{\geq 1} \cdot \Delta_{+}, P_{\mu} \cap P_{\gamma}$ is a closed, nowhere-dense subset of $P_{\mu}$. Hence $P_{\mu} \cap \mathscr{D}$ is dense in $P_{\mu}$ for $0 \neq \mu \in Q_{+} \backslash \mathbb{Z}_{\geq 1} \cdot \Delta_{+}$, because

$$
\mathscr{D}=\left\{\lambda \in \mathfrak{h}^{*} \mid V(\lambda) \text { is irreducible }\right\}=\mathfrak{h}^{*} \backslash \bigcup_{\gamma \in \mathbb{Z}_{\geq 1} \cdot \Delta_{+}} P_{\gamma}
$$

Now the rest of the argument in the proof of [GW, Lemma 4.4] carries over unchanged, by using Lemma 4.3.6 instead of [GW, Corollary 4.3].

We need a few more lemmas for the main result of this subsection. For $\alpha \in W \cdot \Pi^{i m}$, let $\theta_{\alpha, 1} \in U\left(\mathfrak{n}_{-} \oplus \mathfrak{h}\right)_{-\alpha}$ be the element in Theorem 4.3.4. Looking upon $U\left(\mathfrak{n}_{-} \oplus \mathfrak{h}\right)$ as canonically equal to $U\left(\mathfrak{n}_{-}\right) \otimes_{\mathbb{C}} U(\mathfrak{h})$, we define for $\lambda \in \mathfrak{h}^{*}$,

$$
\theta_{\alpha, 1}(\lambda):=(\mathrm{id} \otimes \lambda)\left(\theta_{\alpha, 1}\right) \in U\left(\mathfrak{n}_{-}\right)_{-\alpha} .
$$

It is clear that

$$
\theta_{\alpha, 1} v_{\lambda}=\theta_{\alpha, 1}(\lambda) v_{\lambda}, \quad \text { and } \quad \chi\left(\theta_{\alpha, 1}(\lambda)\right) \equiv 1 \quad\left(\lambda \in \mathfrak{h}^{*}\right)
$$

We put, for $\mu \in Q_{+}$,

$$
q_{\mu}(\lambda):=\chi\left(S_{-\mu}(\lambda)\right) \quad\left(\lambda \in \mathfrak{h}^{*}\right)
$$

Then, as an obvious consequence of Lemma 4.3.5, we have the following. 
Lemma 4.3.8 (cf. [RW, Lemma 3.8]). Let $\mu \in Q_{+} \backslash\{0\}$.

(1) If $y \in U\left(\mathfrak{n}_{-}\right)_{-\mu}$, then $\left(S_{-\mu}(\lambda), y\right)_{\lambda}=\chi(y) R_{\mu}(\lambda)$ for $\lambda \in \mathfrak{h}^{*}$.

(2) $\left(S_{-\mu}(\lambda), S_{-\mu}(\lambda)\right)_{\lambda}=q_{\mu}(\lambda) R_{\mu}(\lambda)$ for $\lambda \in \mathfrak{h}^{*}$.

Now, for $\alpha \in W \cdot \Pi^{i m}$, we set

$V_{\alpha, 1}:=\left\{\lambda \in \mathfrak{h}_{\alpha, 1}^{*} \mid L_{j \beta}(\lambda) \neq 0\right.$ for $\beta \in \Delta_{+}, j \in \mathbb{Z}_{\geq 1}$ such that $\left.j \beta \leq \alpha, j \beta \neq \alpha\right\}$, and

$$
\left(\mathfrak{h}_{\alpha, 1}^{*}\right)^{c}:=\left\{\lambda \in \mathfrak{h}_{\alpha, 1}^{*} \mid L_{\gamma}(\lambda) \neq 0 \text { for } \gamma \in Q_{+} \backslash\{0\} \text { such that } \gamma \neq \alpha\right\} .
$$

Remark 4.3.9. From the assumption that $a_{i i} \neq 0 \quad(i \in I)$, it immediately follows that $V_{\alpha, 1}$ is Zariski-dense in $\mathfrak{h}_{\alpha, 1}^{*}$ and that $\left(\mathfrak{h}_{\alpha, 1}^{*}\right)^{c}$ is not empty.

Lemma 4.3.10 (cf. [RW, Lemma 3.9]). Let $\alpha \in W \cdot \Pi^{i m}$.

(1) If $\lambda \in \mathfrak{h}_{\alpha, 1}^{*}$, then $S_{-\alpha}(\lambda)=q_{\alpha}(\lambda) \theta_{\alpha, 1}(\lambda)$.

(2) If $\lambda \in V_{\alpha, 1}$, then $q_{\alpha}(\lambda) \neq 0$.

Sketch of proof. For the assertion (1), we first note that if $\lambda \in V_{\alpha, 1}$, then by [KK, Proposition 4.1(a)],

$$
\operatorname{dim}_{\mathbb{C}} \operatorname{Hom}_{\mathfrak{g}(A)}(V(\lambda-\alpha), V(\lambda))=1 .
$$

(Recall that $\operatorname{dim}_{\mathbb{C}} \mathfrak{g}_{\alpha}=1$ for $\alpha \in W \cdot \Pi$.) Then the rest of the argument used to prove [RW, Lemma 3.9 (1)] applies to our case.

For the assertion (2), we take an element $\zeta \in \mathfrak{h}^{*}$ such that $(\zeta \mid \eta) \neq 0$ for any $\eta \in Q_{+} \backslash\{0\}$, instead of $\rho \in \mathfrak{h}^{*}$ in the proof of [RW, Lemma 3.9]. Then the proof is exactly the same as that of [RW, Lemma $3.9(2)]$.

Finally, we come to the main part of this subsection. Let $\alpha=\sum_{i \in I} m_{i} \alpha_{i} \in$ $W \cdot \Pi^{i m}, \lambda \in \mathfrak{h}_{\alpha, 1}^{*}$, and let $\theta_{\alpha, 1} \in U\left(\mathfrak{n}_{-} \oplus \mathfrak{h}\right)_{-\alpha}$ be the element in Theorem 4.3.4. Then it easily follows that $\theta_{\alpha, 1}(\lambda) v_{\lambda}=\theta_{\alpha, 1} v_{\lambda} \in V(\lambda)$ is a nonzero highest weight vector of weight $\lambda-\alpha$, where $v_{\lambda}$ is the canonical generator of $V(\lambda)$. Hence we get an embedding

$$
T(\lambda): V(\lambda-\alpha) \hookrightarrow V(\lambda),
$$

where $T(\lambda) v_{\lambda-\alpha}=\theta_{\alpha, 1}(\lambda) v_{\lambda}$ with $v_{\lambda-\alpha}$ the canonical generator of $V(\lambda-\alpha)$. Now we set

$$
N(\lambda):=V(\lambda) / T(\lambda) V(\lambda-\alpha) .
$$

We fix root vectors $f_{n+1}, f_{n+2}, \ldots \in \mathfrak{n}_{-}=\sum_{\alpha \in \Delta_{+}}^{\oplus} \mathfrak{g}_{-\alpha}$ so that $f_{1}, f_{2}, \ldots$ is a basis of $\mathfrak{n}_{-}$by root vectors. We set $\bar{\theta}_{\alpha, 1}:=f_{1}^{m_{1}} \ldots f_{n}^{m_{n}}$. Then, for each $\mu \in Q_{+}$, we can construct a complementary subspace $V_{-\mu}$ to $U\left(\mathfrak{n}_{-}\right)_{-\mu+\alpha} \cdot \bar{\theta}_{\alpha, 1}$ in $U\left(\mathfrak{n}_{-}\right)_{-\mu}$, such that for all $\xi \in \mathfrak{h}^{*}$,

$$
V_{-\mu} \oplus\left(U\left(\mathfrak{n}_{-}\right)_{-\mu+\alpha} \cdot \theta_{\alpha, 1}(\xi)\right)=U\left(\mathfrak{n}_{-}\right)_{-\mu}
$$

as a vector space (see $[\mathrm{S}, \S 3])$. Now we set $V:=\sum_{\mu \in Q_{+}}^{\oplus} V_{-\mu}$.

Then, for $\xi \in \mathfrak{h}_{\alpha, 1}^{*}, V$ is isomorphic to $N(\xi)$ as a vector space via the map $\psi_{\xi}$ given by

$$
\psi_{\xi}(Y)=Y v_{\xi}+T(\xi) V(\xi-\alpha) \quad(Y \in V) .
$$


Therefore, via the above map $\psi_{\xi}$, we may look upon $V$ as a $\mathfrak{g}(A)$-module with an action $\pi_{\xi}$ of $\mathfrak{g}(A)$. (Here we remark that, for $Y \in V_{-\mu}\left(\mu \in Q_{+}\right)$and $h \in \mathfrak{h}$, we have $\pi_{\xi}(h) Y=\langle\xi-\mu, h\rangle Y$.)

On the other hand, the Shapovalov form $(\cdot, \cdot)_{\xi}$ induces a contravariant bilinear form (again denoted by $(\cdot, \cdot)_{\xi}$ ) on $N(\xi)=V(\xi) / T(\xi) V(\xi-\alpha)$, since $T(\xi) V(\xi-\alpha)$ is obviously contained in the radical of the Shapovalov form on $V(\xi)$. So, pulling back this form $(\cdot, \cdot)_{\xi}$ on $N(\xi)$ by $\psi_{\xi}$, we can define a symmetric, $\pi_{\xi}$-contravariant bilinear form $\langle\cdot \mid \cdot\rangle_{\xi}$ on $V$ (depending on $\xi \in$ $\left.\mathfrak{h}_{\alpha, 1}^{*}\right)$.

Later, we shall need the following lemma.

Lemma 4.3.11. Let $\xi \in\left(\mathfrak{h}_{\alpha, 1}^{*}\right)^{c}$. Then the form $\langle\cdot \mid \cdot\rangle_{\xi}$ on $V$ is nondegenerate.

Proof. We show that $(\cdot, \cdot)_{\xi}$ on $N(\xi)$ is nondegenerate. This is equivalent to the irreducibility of $N(\xi)$, since $N(\xi)$ is a highest weight $\mathfrak{g}(A)$-module with highest weight $\xi$. Hence it suffices to show that if, for some $\eta \in Q_{+}$, there exists a nonzero highest weight vector $\bar{v} \in N(\xi)_{\xi-\eta}$, then $\eta=0$.

Suppose that $\eta \neq 0$. Then it readily follows from the definition of the Casimir operator $\Omega$ and the fact that $\Omega$ acts on $N(\xi)$ by the scalar $(\xi+\rho \mid \xi+\rho)$ $-(\rho \mid \rho)$ that

$$
\begin{aligned}
C \bar{v} & =((\xi+\rho \mid \xi+\rho)-(\rho \mid \rho)) \bar{v} \\
& =((\xi-\eta+\rho \mid \xi-\eta+\rho)-(\rho \mid \rho)) \bar{v} .
\end{aligned}
$$

So we get $2(\xi+\rho \mid \eta)=(\eta \mid \eta)$. Therefore, from the assumption of the lemma, it follows that $\eta=\alpha$.

Thus we obtain a nonzero weight vector $v \in V(\xi)_{\xi-\alpha}$ of weight $\xi-\alpha$ such that $v \notin T(\xi) V(\xi-\alpha)$ and $\mathfrak{n}_{+} v \in T(\xi) V(\xi-\alpha)$. Now it is obvious that $\mathrm{n}_{+} v=0$. So we have got two linearly independent embeddings of $V(\xi-\alpha)$ into $V(\xi)$. This is a contradiction, since we have $\operatorname{dim}_{\mathbb{C}} \operatorname{Hom}_{\mathfrak{g}(A)}(V(\xi-\alpha), V(\xi))=1$ by [KK, Proposition 4.1(a)]. This proves the lemma.

We fix an element $\alpha=w\left(\alpha_{j}\right) \quad\left(w \in W, \alpha_{j} \in \Pi^{i m}\right)$ of $W \cdot \Pi^{i m}$, and an element $\lambda \in \mathfrak{h}_{\alpha, 1}^{*}$. Let $\omega \in \mathfrak{h}^{*}$ be an element such that $(\omega \mid \alpha)=0$ and $(\omega \mid \beta) \neq 0 \quad\left(\beta \in \Delta_{+} \backslash\{\alpha\}\right)$.

Now we give a construction of the desired filtration of $N(\lambda)=V(\lambda) /$ $T(\lambda) V(\lambda-\alpha)$ by $\mathfrak{g}(A)$-submodules. We look upon $\left.\mathbb{C}[t] \otimes_{\mathbb{C}} V=\sum_{\mu \in Q_{+}}^{\oplus} \mathbb{C} t\right] \otimes_{\mathbb{C}}$ $V_{-\mu}$ as the direct sum of the spaces of all polynomials in one variable $t$ with values in $V_{-\mu}\left(\mu \in Q_{+}\right)$. Set, for each $i \in \mathbb{Z}_{\geq 0}$,

$N(\lambda)_{i}:=\left\{\psi_{\lambda}(f(0)) \in N(\lambda) \mid f \in \mathbb{C}[t] \otimes_{\mathbb{C}} V,\langle f(t) \mid v\rangle_{\lambda+t \omega} \in t^{i} \mathbb{C}[t]\right.$ for all $\left.v \in V\right\}$.

Then we get a filtration (called Jantzen's filtration)

$$
N(\lambda)=N(\lambda)_{0} \supset N(\lambda)_{1} \supset N(\lambda)_{2} \supset \cdots
$$

of $N(\lambda)$ by $\mathfrak{g}(A)$-submodules. Here $N(\lambda) / N(\lambda)_{1}$ is isomorphic to $L(\lambda)$ as a $\mathfrak{g}(A)$-module. Furthermore, the following theorem $(=$ the first assertion of Theorem II) holds.

Theorem 4.3.12 (cf. [RW, Theorem 3.10]). Let $\mathfrak{g}(A)$ be a GKM algebra associated to a symmetrizable $G G C M A=\left(a_{i j}\right)_{i, j \in I}$ satisfying the condition that 
$a_{i i} \neq 0 \quad(i \in I)$. Let $\alpha=w\left(\alpha_{j}\right) \in W \cdot \Pi^{i m}$ with $w \in W$ and $\alpha_{j} \in \Pi^{i m}$, and let $\lambda \in \mathfrak{h}_{\alpha, 1}^{*}$. Then, for the filtration

$$
N(\lambda)=N(\lambda)_{0} \supset N(\lambda)_{1} \supset N(\lambda)_{2} \supset \cdots
$$

of $N(\lambda)$ given above, the following equality holds in the algebra $\mathscr{E}$ :

$$
\begin{aligned}
\sum_{i \geq 1} \operatorname{ch} N(\lambda)_{i}= & \sum_{\beta \in \Delta_{+}} \sum_{\substack{l \in \mathbb{Z}_{\geq 1} \\
L_{l \beta}(\lambda)=0}} \operatorname{ch} V(\lambda-l \beta) \\
& -\sum_{\gamma \in \Delta_{+}} \sum_{\substack{m \in \mathbb{Z}_{\geq 1} \\
L_{m \gamma}(\lambda-\alpha)=0}} \operatorname{ch} V(\lambda-\alpha-m \gamma)-a(\lambda) \operatorname{ch} V(\lambda-\alpha),
\end{aligned}
$$

where $a(\lambda) \in \mathbb{Z}$, the roots $\beta \in \Delta_{+}$and $\gamma \in \Delta_{+}$are taken with their multiplicities. Sketch of proof. Let $\mu \in Q_{+}$. Fix a basis $\left\{v_{1}, \ldots, v_{d_{\mu}}\right\}$ of $V_{-\mu}\left(\subset U\left(\mathfrak{n}_{-}\right)_{-\mu}\right)$. We set, for $\xi \in \mathfrak{h}_{\alpha, 1}^{*}$,

$$
D_{\xi-\mu}^{\prime}(\xi):=\operatorname{det}\left(\left\langle v_{i} \mid v_{j}\right\rangle_{\xi}\right)_{1 \leq i, j \leq d_{\mu}}=\operatorname{det}\left(\left(v_{i}, v_{j}\right)_{\xi}\right)_{1 \leq i, j \leq d_{\mu}} .
$$

It is clear that $\lambda+t \omega \in \mathfrak{h}_{\alpha, 1}^{*}$ for all $t \in \mathbb{C}$. We note that if $\lambda+t_{0} \omega$ $\in\left(\mathfrak{h}_{\alpha, 1}^{*}\right)^{c}(\neq \varnothing)$ for some $t_{0} \in \mathbb{C}$, then the form $\langle\cdot \mid \cdot\rangle_{\lambda+t_{0} \omega}$ is nondegenerate on $V=\sum_{\mu \in Q_{+}}^{\oplus} V_{-\mu}$ by Lemma 4.3.11, and so $D_{\lambda+t_{0} \omega-\mu}^{\prime}\left(\lambda+t_{0} \omega\right)=$ $\operatorname{det}\left(\left\langle v_{i} \mid v_{j}\right\rangle_{\lambda+t_{0} \omega}\right)_{1 \leq i, j \leq d_{\mu}} \neq 0$ for all $\mu \in Q_{+}$. Since such $t_{0} \in \mathbb{C}$ clearly exists, the polynomial map $D_{\lambda+t \omega-\mu}^{\prime}(\lambda+t \omega)$ in $t$ is not identically equal to zero. Hence, for each $\mu \in Q_{+}$, there exists some $\varepsilon=\varepsilon(\mu)>0$ such that $D_{\lambda+t \omega-\mu}^{\prime}(\lambda+t \omega) \neq 0$ for all $t \in \mathbb{C}$ with $0<|t|<\varepsilon$.

Then, using [J, Lemma 5.1] applied to $\mathbb{C}[t] \otimes_{\mathbb{C}} V_{-\mu}$, we can easily deduce that

$$
\operatorname{ord}_{0}\left(D_{\lambda+t \omega-\mu}^{\prime}(\lambda+t \omega)\right)=\sum_{i \geq 1} \operatorname{dim}_{\mathbb{C}}\left(N(\lambda)_{i} \cap N(\lambda)_{\lambda-\mu}\right),
$$

where $\operatorname{ord}_{0}(f(t)) \quad(f(t) \neq 0 \in \mathbb{C}[t])$ denotes the largest power of $t$ dividing $f(t)$. Thus, we obtain

$$
\sum_{i \geq 1} \operatorname{ch} N(\lambda)_{i}=e(\lambda) \cdot \sum_{\mu \in Q_{+}} \operatorname{ord}_{0}\left(D_{\lambda+t \omega-\mu}^{\prime}(\lambda+t \omega)\right) e(-\mu) .
$$

Hence we have only to compute $\operatorname{ord}_{0}\left(D_{\lambda+t \omega-\mu}^{\prime}(\lambda+t \omega)\right)$ for $\mu \in Q_{+}$.

By the same argument as in the proof of [RW, Theorem 3.10], using Theorem 4.1.1, we obtain

$$
\begin{aligned}
\operatorname{ord}_{0}\left(D_{\lambda+t \omega-\mu}^{\prime}(\lambda+t \omega)\right) & \\
= & \sum_{\beta \in \Delta_{+}} \sum_{\substack{l \in \mathbb{Z}_{\geq 1} \\
L_{l \beta}(\lambda)=0}} K(\mu-l \beta)-\sum_{\gamma \in \Delta_{+}} \sum_{\substack{m \in \mathbb{Z}_{\geq 1} \\
L_{m \gamma}(\lambda-\alpha)=0}} K(\mu-\alpha-m \gamma) \\
& \quad+K(\mu-\alpha) \cdot \operatorname{ord}_{0}\left(q_{\alpha}(\lambda+t \omega)\right)-K(\mu-\alpha) \cdot \operatorname{ord}_{0}\left(R_{\alpha}(\lambda+t \omega)\right),
\end{aligned}
$$

where the roots $\beta \in \Delta_{+}$and $\gamma \in \Delta_{+}$are taken with their multiplicities, and $K(\eta) \quad\left(\eta \in Q_{+}\right)$is the generalized Kostant partition function. (We have also used the assumption that $a_{i i} \neq 0 \quad(i \in I)$ in this computation.) 
Here we should mention the following misprints in the proof of [RW, Theorem 3.10]: the determinant $D_{\xi-k \alpha-\mu}(\xi-k \alpha)$ in the equation (3.12) should be replaced by $D_{(\xi-k \alpha)-(\mu-k \alpha)}(\xi-k \alpha)$, and similarly, $D_{\nu-k \alpha-\mu}(\nu-k \alpha)$ in the equation (3.13) should be replaced by $D_{(\nu-k \alpha)-(\mu-k \alpha)}(\nu-k \alpha)$ (in our case, $k=1)$.

Now we recall that for $\tau \in \mathfrak{h}^{*}$,

$$
\operatorname{ch} V(\tau)=e(\tau) \cdot \sum_{\eta \in Q_{+}} K(\eta) e(-\eta) .
$$

Then, from the identities (4.3.2), (4.3.3), and (4.3.4), we can easily derive the desired formula (4.3.1) in the algebra $\mathscr{E}$. This completes the proof of the theorem.

The formula (4.3.1) in Theorem 4.3.12 is called Jantzen's character sum formula.

\section{SOME FURTHER INFORMATION ABOUT THE CONSTANT $a(\lambda)$}

Let $\Lambda \in P_{+}, w \in W$, and $\alpha_{j} \in \Pi^{i m}$ with $\left(\Lambda \mid \alpha_{j}\right)=0$. We put $\lambda:=$ $w(\Lambda+\rho)-\rho \in \mathfrak{h}^{*}, \alpha:=w\left(\alpha_{j}\right) \in W \cdot \Pi^{i m} \subset \Delta_{+}$. Then we have

$$
\begin{aligned}
2(\lambda+\rho \mid \alpha)-(\alpha \mid \alpha) & =2\left(w(\Lambda+\rho) \mid w\left(\alpha_{j}\right)\right)-\left(w\left(\alpha_{j}\right) \mid w\left(\alpha_{j}\right)\right) \\
& =2\left(\Lambda+\rho \mid \alpha_{j}\right)-\left(\alpha_{j} \mid \alpha_{j}\right) \\
& =2\left(\rho \mid \alpha_{j}\right)-\left(\alpha_{j} \mid \alpha_{j}\right) \\
& =0 .
\end{aligned}
$$

Thus we have $\lambda \in \mathfrak{h}_{\alpha, 1}^{*}$, and hence we can apply Theorem 4.3.12 to this case.

In this section, we first apply Theorem 4.2.1 to obtain that $[V((w, 0) \circ \Lambda)$ : $\left.L\left(\left(w, \alpha_{j}\right) \circ \Lambda\right)\right]=1$. Then we apply Theorem 4.3.12 to show that the constant $a(\lambda)$ in Theorem 4.3.12 is equal to 1 in the above case (which is the second assertion of Theorem II). The latter fact plays an important role in the proof of the Kazhdan-Lusztig conjecture in $\S 6$.

5.1. Application of the character sum formula for a Verma module. Now we shall make use of Jantzen's character sum formula for a single Verma module to compute the multiplicity $\left[V((w, 0) \circ \Lambda): L\left(\left(w, \alpha_{j}\right) \circ \Lambda\right)\right]$. Let $\mathfrak{g}(A)$ be a GKM algebra associated to a symmetrizable GGCM $A=\left(a_{i j}\right)_{i, j \in I}$ with $a_{i i} \neq 0$ $(i \in I)$. Note that, in this case, the set $\mathscr{A}(\Lambda)$ consists of all sums of distinct, pairwise perpendicular, imaginary simple roots perpendicular to $\Lambda \in P_{+}$. In particular, $\mathscr{A}(\Lambda)$ is a finite set.

Theorem 5.1.1. Let $\Lambda \in P_{+}, w \in W$, and $\beta, \beta^{\prime} \in \mathscr{A}(\Lambda)$. If $\beta^{\prime} \geqslant \beta$ and $\operatorname{ht}\left(\beta^{\prime}\right)=\operatorname{ht}(\beta)+1$, then we have $\left[V((w, \beta) \circ \Lambda): L\left(\left(w, \beta^{\prime}\right) \circ \Lambda\right)\right]=1$.

Proof. First we note that by the assumption of the theorem, there exists $\alpha_{j} \in$ $\Pi^{i m}$ with $\left(\Lambda \mid \alpha_{j}\right)=0,\left(\beta \mid \alpha_{j}\right)=0$ such that $\beta^{\prime}=\beta+\alpha_{j}$. So we have

$$
w\left(\Lambda+\rho-\beta^{\prime}\right)-\rho=w\left((\Lambda-\beta)+\rho-\alpha_{j}\right)-\rho,
$$

where $\Lambda-\beta \in P_{+},\left(\Lambda-\beta \mid \alpha_{j}\right)=0$. Hence we may assume that $\beta=0$, and that $\operatorname{ht}\left(\beta^{\prime}\right)=1$. 
Put $\lambda:=w(\Lambda+\rho)-\rho \in \mathfrak{h}^{*}, \mu:=w\left(\Lambda+\rho-\alpha_{j}\right)-\rho=\left(w, \alpha_{j}\right) \circ \Lambda$. Then, by Theorem 4.2.1, the Verma module $V(\lambda)$ has a filtration

$$
V(\lambda) \supset V(\lambda)_{1} \supset V(\lambda)_{2} \supset \cdots
$$

by $\mathfrak{g}(A)$-submodules such that $V(\lambda) / V(\lambda)_{1} \cong L(\lambda)$, and such that

$$
\sum_{i \geq 1} \operatorname{ch} V(\lambda)_{i}=\sum_{\beta \in \Delta_{+}} \sum_{\substack{l \in \mathbb{Z}_{\geq 1} \\ 2(\lambda+\rho \mid \beta)=l(\beta \mid \beta)}} \operatorname{ch} V(\lambda-l \beta) .
$$

Since we have the exact sequence

$$
0 \longrightarrow V(\lambda)_{1} \longrightarrow V(\lambda) \longrightarrow L(\lambda) \longrightarrow 0,
$$

we get

$$
[V(\lambda): L(\mu)]=\left[V(\lambda)_{1}: L(\mu)\right]+[L(\lambda): L(\mu)] .
$$

Here we recall that, for $\Lambda \in P_{+}$and $\left(w_{1}, \beta_{1}\right),\left(w_{2}, \beta_{2}\right) \in W \times \mathscr{A}(\Lambda),\left(w_{1}, \beta_{1}\right) \circ$ $\Lambda=\left(w_{2}, \beta_{2}\right) \circ \Lambda$ if and only if $\left(w_{1}, \beta_{1}\right)=\left(w_{2}, \beta_{2}\right)$, since $\left\langle\Lambda+\rho-\beta_{i}, \alpha_{k}^{\vee}\right\rangle \in$ $\mathbb{Z}_{>1}(i=1,2)$ for $k \in I^{r e}$ (cf. the proof of [K, Proposition 3.12]). So we obtain $[L(\lambda): L(\mu)]=0$. Thus, we have to show that $\left[V(\lambda)_{1}: L(\mu)\right]=1$.

Note that, in the formula (5.1.1), all $\operatorname{ch} V(\lambda)_{i}(i \geq 1)$ on the left-hand side, and all $\operatorname{ch} V(\lambda-l \beta) \quad\left(\beta \in \Delta_{+}, l \in \mathbb{Z}_{\geq 1}\right)$ on the right-hand side are clearly (possibly infinite) sums of some scalar multiple of $\operatorname{ch} L(\lambda-\eta) \quad\left(\eta \in Q_{+}\right)$by Proposition 3.2.1. Furthermore, $\operatorname{ch} L(\lambda-\eta) \quad\left(\eta \in Q_{+}\right)$are linearly independent over $\mathbb{C}$ (i.e., $\sum_{\eta \in Q_{+}} a_{\eta} \operatorname{ch} L(\lambda-\eta)=0$ with $a_{\eta} \in \mathbb{C}$ implies $a_{\eta}=0$ for all $\left.\eta \in Q_{+}\right)$. Therefore, we have that

$$
\left[V(\lambda)_{1}: L(\mu)\right] \geq\left[V(\lambda)_{2}: L(\mu)\right] \geq \cdots,
$$

and that

$$
\sum_{i \geq 1}\left[V(\lambda)_{i}: L(\mu)\right]=\sum_{\beta \in \Delta_{+}} \sum_{\substack{l \in \mathbb{Z}_{\geq 1} \\ 2(\lambda+\rho \mid \beta)=l(\beta \mid \beta)}}[V(\lambda-l \beta): L(\mu)] .
$$

Let us consider the equation

$$
2(\lambda+\rho \mid \beta)=l(\beta \mid \beta),
$$

or equivalently,

$$
2(w(\Lambda+\rho) \mid \beta)=l(\beta \mid \beta) \quad\left(\beta \in \Delta_{+}, l \in \mathbb{Z}_{\geq 1}\right) .
$$

By Lemma 4.3.1, the above equation is equivalent to either of the following:

(a) $\beta \in \Delta_{+} \cap \Delta^{r e}$ and $r_{\beta} w \geqslant w$;

(b) $l=1$, and $w^{-1}(\beta) \in \Pi^{i m}$ with $\left(\Lambda \mid w^{-1}(\beta)\right)=0$.

(Note that in both cases, we have $\operatorname{dim}_{\mathbb{C}} \mathfrak{g}_{\beta}=1$.)

In case $(\mathrm{a})$, we have $l=\left\langle w(\Lambda+\rho), \beta^{\vee}\right\rangle$, so that

$$
\begin{aligned}
\lambda-l \beta & =w(\Lambda+\rho)-\rho-\left\langle w(\Lambda+\rho), \beta^{\vee}\right\rangle \beta \\
& =r_{\beta}(w(\Lambda+\rho))-\rho \\
& =\left(r_{\beta} w, 0\right) \circ \Lambda .
\end{aligned}
$$

In this case, we have

$$
[V(\lambda-l \beta): L(\mu)]=\left[V\left(\left(r_{\beta} w, 0\right) \circ \Lambda\right): L\left(\left(w, \alpha_{j}\right) \circ \Lambda\right)\right]=0,
$$


since, by Theorem 3.3.3, $\left[V\left(\left(r_{\beta} w, 0\right) \circ \Lambda\right): L\left(\left(w, \alpha_{j}\right) \circ \Lambda\right)\right] \neq 0$ implies $\left(w, \alpha_{j}\right) \geqslant\left(r_{\beta} w, 0\right)$. This contradicts the assumption that $r_{\beta} w \geqslant w$.

In case (b), we have, for some $\alpha_{k}:=w^{-1}(\beta) \in \Pi^{i m}$ with $\left(\Lambda \mid \alpha_{k}\right)=0$,

$$
\begin{aligned}
\lambda-l \beta & =\lambda-\beta \\
& =w(\Lambda+\rho)-\rho-w\left(\alpha_{k}\right) \\
& =w\left(\Lambda+\rho-\alpha_{k}\right)-\rho \\
& =\left(w, \alpha_{k}\right) \circ \Lambda .
\end{aligned}
$$

In this case, we have, by Theorem 3.3.3,

$$
\begin{aligned}
{[V(\lambda-l \beta): L(\mu)] } & =\left[V\left(\left(w, \alpha_{k}\right) \circ \Lambda\right): L\left(\left(w, \alpha_{j}\right) \circ \Lambda\right)\right] \\
& = \begin{cases}1 & \text { if } \alpha_{k}=\alpha_{j}, \\
0 & \text { otherwise. }\end{cases}
\end{aligned}
$$

To sum up, we have obtained

$$
\sum_{i \geq 1}\left[V(\lambda)_{i}: L(\mu)\right]=1 .
$$

Therefore, we get $\left[V(\lambda)_{1}: L(\mu)\right]=1,\left[V(\lambda)_{i}: L(\mu)\right]=0 \quad(i \geq 2)$. Thus, we have proved the theorem.

5.2. The constant $a(\lambda)$. To show the second assertion of Theorem II, again we compute the multiplicity $\left[V((w, 0) \circ \Lambda): L\left(\left(w, \alpha_{j}\right) \circ \Lambda\right)\right]=[V(\lambda): L(\mu)]$ above, this time by using Theorem 4.3.12. Let $\Lambda \in P_{+}$, and let $\alpha=w\left(\alpha_{j}\right) \in W \cdot \Pi^{i m}$ with $\left(\Lambda \mid \alpha_{j}\right)=0$. Then $\lambda=w(\Lambda+\rho)-\rho \in \mathfrak{h}_{\alpha, 1}^{*}$, as shown before. So, since

$$
\begin{aligned}
\lambda-\alpha & =w(\Lambda+\rho)-\rho-w\left(\alpha_{j}\right) \\
& =w\left(\Lambda+\rho-\alpha_{j}\right)-\rho \\
& =\left(w, \alpha_{j}\right) \circ \Lambda \\
& =\mu,
\end{aligned}
$$

we have an embedding

$$
T(\lambda): V(\mu)=V\left(\left(w, \alpha_{j}\right) \circ \Lambda\right) \hookrightarrow V((w, 0) \circ \Lambda)=V(\lambda) .
$$

Therefore, we obtain

$$
\begin{aligned}
1 & =[V(\lambda): L(\mu)] \\
& =\left[V\left(\left(w, \alpha_{j}\right) \circ \Lambda\right): L\left(\left(w, \alpha_{j}\right) \circ \Lambda\right)\right]+\left[V(\lambda) / T(\lambda) V(\lambda-\alpha): L\left(\left(w, \alpha_{j}\right) \circ \Lambda\right)\right],
\end{aligned}
$$

and hence

$$
\left[V(\lambda) / T(\lambda) V(\lambda-\alpha): L\left(\left(w, \alpha_{j}\right) \circ \Lambda\right)\right]=0 .
$$

Using this fact, we get the following proposition $(=$ the second assertion of Theorem II).

Proposition 5.2.1. Let $\mathfrak{g}(A)$ be a $G K M$ algebra associated to a symmetrizable GGCM $A=\left(a_{i j}\right)_{i, j \in I}$ with $a_{i i} \neq 0(i \in I)$. Let $\Lambda \in P_{+}, w \in W$, and $\alpha_{j} \in$ $\Pi^{\text {im }}$ with $\left(\Lambda \mid \alpha_{j}\right)=0$. Put $\lambda:=w(\Lambda+\rho)-\rho \in \mathfrak{h}^{*}$, and $\alpha:=w\left(\alpha_{j}\right) \in W \cdot \Pi^{i m}$. Then $\lambda \in \mathfrak{h}_{\alpha, 1}^{*}$. Moreover, in this case, the constant $a(\lambda)$ in the formula (4.3.1) in Theorem 4.3 .12 is equal to 1 .

Proof. Put $N(\lambda):=V(\lambda) / T(\lambda) V(\lambda-\alpha)$ and $\mu:=\left(w, \alpha_{j}\right) \circ \Lambda$. Then we have $[N(\lambda): L(\mu)]=0$, as seen above. So we have $\left[N(\lambda)_{i}: L(\mu)\right]=0 \quad(i \geq 1)$. 
Note that, in formula (4.3.1) in Theorem 4.3.12, all $\operatorname{ch} N(\lambda)_{i} \quad(i \geq 1)$ on the left-hand side, and all $\operatorname{ch} V(\lambda-l \beta)\left(\beta \in \Delta_{+}, l \in \mathbb{Z}_{\geq 1}\right), \operatorname{ch} V(\lambda-\alpha-m \gamma)$ $\left(\gamma \in \Delta_{+}, m \in \mathbb{Z}_{\geq 1}\right)$ on the right-hand side are clearly (possibly infinite) sums of some scalar multiple of $\operatorname{ch} V(\lambda-\eta)\left(\eta \in Q_{+}\right)$by Proposition 3.2.1. Furthermore, $\operatorname{ch} L(\lambda-\eta) \quad\left(\eta \in Q_{+}\right)$are linearly independent over $\mathbb{C}$ (see the proof of Theorem 5.1.1).

Therefore, we have

$$
\begin{aligned}
0= & \sum_{i \geq 1}\left[N(\lambda)_{i}: L(\mu)\right] \\
= & \sum_{\beta \in \Delta_{+}} \sum_{\substack{l \in \mathbb{Z}_{\geq 1} \\
L_{l \beta}(\lambda)=0}}[V(\lambda-l \beta): L(\mu)]-\sum_{\gamma \in \Delta_{+}} \sum_{\substack{m \in \mathbb{Z}_{\geq 1} \\
L_{m \gamma}(\lambda-\alpha)=0}}[V(\lambda-\alpha-m \gamma): L(\mu)] \\
& \quad-a(\lambda)[V(\lambda-\alpha): L(\mu)] .
\end{aligned}
$$

It has already been seen in the proof of Theorem 5.1.1 that

$$
\sum_{\beta \in \Delta_{+}} \sum_{\substack{l \in \mathbb{Z}_{\geq 1} \\ L_{l \beta}(\lambda)=0}}[V(\lambda-l \beta): L(\mu)]=1 .
$$

Now consider the equation

$$
L_{m \gamma}(\lambda-\alpha)=0,
$$

or equivalently,

$$
2\left(w\left(\Lambda+\rho-\alpha_{j}\right) \mid \gamma\right)=m(\gamma \mid \gamma) \quad\left(\gamma \in \Delta_{+}, m \in \mathbb{Z}_{\geq 1}\right) .
$$

By Lemma 4.3.1, the above equation is equivalent to either of the following:

(a) $\gamma \in \Delta_{+} \cap \Delta^{r e}$ and $r_{\gamma} w \geqslant w$;

(b) $m=1$, and $w^{-1}(\gamma) \in \Pi^{i m}$ with $\left(\Lambda-\alpha_{j} \mid w^{-1}(\gamma)\right)=0$.

(Note that in both cases, we have $\operatorname{dim}_{\mathbb{C}} \mathfrak{g}_{\gamma}=1$.)

In case (a), we have $m=\left\langle w\left(\Lambda+\rho-\alpha_{j}\right), \gamma^{\vee}\right\rangle$, so that

$$
\begin{aligned}
\lambda-\alpha-m \gamma & =w(\Lambda+\rho)-\rho-w\left(\alpha_{j}\right)-m \gamma \\
& =w\left(\Lambda+\rho-\alpha_{j}\right)-m \gamma-\rho \\
& =r_{\gamma}\left(w\left(\Lambda+\rho-\alpha_{j}\right)\right)-\rho \\
& =\left(r_{\gamma} w, \alpha_{j}\right) \circ \Lambda .
\end{aligned}
$$

In this case, we have, by Theorem 3.3.3,

$$
[V(\lambda-\alpha-m \gamma): L(\mu)]=\left[V\left(\left(r_{\gamma} w, \alpha_{j}\right) \circ \Lambda\right): L\left(\left(w, \alpha_{j}\right) \circ \Lambda\right)\right]=0,
$$

since $r_{\gamma} w \geqslant w$.

In case (b), we have, for some $\alpha_{k}:=w^{-1}(\gamma) \in \Pi^{i m}$ with $\left(\Lambda-\alpha_{j} \mid \alpha_{k}\right)=0$,

$$
\begin{aligned}
\lambda-\alpha-m \gamma & =w(\Lambda+\rho)-\rho-w\left(\alpha_{j}\right)-w\left(\alpha_{k}\right) \\
& =w\left(\Lambda+\rho-\left(\alpha_{j}+\alpha_{k}\right)\right)-\rho \\
& =\left(w, \alpha_{j}+\alpha_{k}\right) \circ \Lambda .
\end{aligned}
$$

Here note that $\alpha_{j}+\alpha_{k} \in \mathscr{A}(\Lambda)$. In this case, we have, by Theorem 3.3.3,

$$
[V(\lambda-\alpha-m \gamma): L(\mu)]=\left[V\left(\left(w, \alpha_{j}+\alpha_{k}\right) \circ \Lambda\right): L\left(\left(w, \alpha_{j}\right) \circ \Lambda\right)\right]=0 .
$$


Summarizing, we obtain

$$
\begin{aligned}
0 & =1-a(\lambda)[V(\lambda-\alpha): L(\mu)]=1-a(\lambda)\left[V\left(\left(w, \alpha_{j}\right) \circ \Lambda\right): L\left(\left(w, \alpha_{j}\right) \circ \Lambda\right)\right] \\
& =1-a(\lambda),
\end{aligned}
$$

and hence $a(\lambda)=1$. Thus the proposition has been proved.

\section{Proof of THe CONJECTURE}

Fix $\Lambda \in P_{+}$. In this section, we shall prove the Kazhdan-Lusztig conjecture for GKM algebras $\mathfrak{g}(A)$ associated to a symmetrizable GGCM $A=$ $\left(a_{i j}\right)_{i, j \in I}$ with $a_{i i} \neq 0 \quad(i \in I)$, that is, that the multiplicity $[V((w, \beta) \circ \Lambda)$ : $\left.L\left(\left(w^{\prime}, \beta^{\prime}\right) \circ \Lambda\right)\right]$ for $(w, \beta),\left(w^{\prime}, \beta^{\prime}\right) \in W \times \mathscr{A}(\Lambda)$ is equal to $P_{(w, \beta),\left(w^{\prime}, \beta^{\prime}\right)}(1)$, where $P_{(w, \beta),\left(w^{\prime}, \beta^{\prime}\right)}(q)$ is the extended Kazhdan-Lusztig polynomial introduced in $\S 2$.

Furthermore, we shall describe the (formal) character of the irreducible highest weight $\mathfrak{g}(A)$-module $L((w, \beta) \circ \Lambda)$ with highest weight $(w, \beta) \circ \Lambda$ for $(w, \beta) \in W \times \mathscr{A}(\Lambda)$ in terms of the extended inverse Kazhdan-Lusztig polynomials $Q_{(w, \beta),\left(w^{\prime}, \beta^{\prime}\right)}(q) \quad\left(\left(w^{\prime}, \beta^{\prime}\right) \in W \times \mathscr{A}(\Lambda)\right)$.

6.1. Proof of the multiplicity formula. In [N], we proved the following theorem (without assuming that $\left.a_{i i} \neq 0 \quad(i \in I)\right)$.

Theorem 6.1.1 ([N, Theorem 5.3]). Let $\mathfrak{g}(A)$ be a GKM algebra associated to a symmetrizable $G G C M A=\left(a_{i j}\right)_{i, j \in I}$. Let $\Lambda \in P_{+}$, and $\left(w, \beta_{1}\right),\left(z, \beta_{2}\right) \in$ $W \times \mathscr{A}(\Lambda)$. Then we have

$$
\left[V\left(\left(w, \beta_{1}\right) \circ \Lambda\right): L\left(\left(z, \beta_{2}\right) \circ \Lambda\right)\right] \geq P_{\left(w, \beta_{1}\right),\left(z, \beta_{2}\right)}(1),
$$

where $P_{\left(w, \beta_{1}\right),\left(z, \beta_{2}\right)}(q)$ is the extended Kazhdan-Lusztig polynomial. Moreover, the equality holds if $\beta_{1}=\beta_{2}$ or if $w=z=1$.

Furthermore, we conjectured that, under the assumption that $a_{i i} \neq 0 \quad(i \in I)$, the equality holds for any $\left(w, \beta_{1}\right),\left(z, \beta_{2}\right) \in W \times \mathscr{A}(\Lambda)$ in the above theorem.

Now we are ready to prove this conjecture.

Theorem 6.1.2 (multiplicity formula). Let $\mathfrak{g}(A)$ be a GKM algebra associated to a symmetrizable GGCM $A=\left(a_{i j}\right)_{i, j \in I}$ satisfying the condition that $a_{i i} \neq 0$ $(i \in I)$. Let $\Lambda \in P_{+}$, and $\left(w, \beta_{1}\right),\left(z, \beta_{2}\right) \in W \times \mathscr{A}(\Lambda)$. Then we have

$$
\left[V\left(\left(w, \beta_{1}\right) \circ \Lambda\right): L\left(\left(z, \beta_{2}\right) \circ \Lambda\right)\right]=P_{\left(w, \beta_{1}\right),\left(z, \beta_{2}\right)}(1),
$$

where $P_{\left(w, \beta_{1}\right),\left(z, \beta_{2}\right)}(q)$ is the extended Kazhdan-Lusztig polynomial.

Proof. By Theorem 3.3.3 and the definition of the extended Kazhdan-Lusztig polynomials, it suffices to show that, for $\left(z, \beta_{2}\right) \geqslant\left(w, \beta_{1}\right) \in W \times \mathscr{A}(\Lambda)$,

$$
\left[V\left(\left(w, \beta_{1}\right) \circ \Lambda\right): L\left(\left(z, \beta_{2}\right) \circ \Lambda\right)\right]=P_{w, z}(1),
$$

where $P_{w, z}(q)$ is the usual Kazhdan-Lusztig polynomial (associated to $w, z \in$ $W$ ) for the Weyl group $W$. We give a proof of this by double induction on $\operatorname{ht}\left(\beta_{2}\right)-\operatorname{ht}\left(\beta_{1}\right)$ and $\ell(z)-\ell(w)$. When $\operatorname{ht}\left(\beta_{2}\right)-\operatorname{ht}\left(\beta_{1}\right)=0$, the assertion is true by Theorem 6.1.1. When $\ell(z)-\ell(w)=0$, the assertion can be proved by exactly the same argument as the one below. So we omit its proof. 
Now assume that $\operatorname{ht}\left(\beta_{2}\right)-\operatorname{ht}\left(\beta_{1}\right)=t \geq 1, \ell(z)-\ell(w)=s \geq 1$. We first note that

$$
\begin{aligned}
\left(z, \beta_{2}\right) \circ \Lambda & =z\left(\Lambda+\rho-\beta_{2}\right)-\rho \\
& =z\left(\left(\Lambda-\beta_{1}\right)+\rho-\left(\beta_{2}-\beta_{1}\right)\right)-\rho \\
& =\left(z, \beta_{2}-\beta_{1}\right) \circ\left(\Lambda-\beta_{1}\right),
\end{aligned}
$$

where $\Lambda-\beta_{1} \in P_{+}, \beta_{2}-\beta_{1} \in \mathscr{A}\left(\Lambda-\beta_{1}\right)$, and that

$$
\begin{aligned}
\left(w, \beta_{1}\right) \circ \Lambda & =w\left(\Lambda+\rho-\beta_{1}\right)-\rho \\
& =(w, 0) \circ\left(\Lambda-\beta_{1}\right) .
\end{aligned}
$$

Therefore, we may assume that $\beta_{1}=0$.

Since $\operatorname{ht}\left(\beta_{2}\right)=t \geq 1$, we can write $\beta_{2}=\beta^{\prime}+\alpha_{j}$ for some $\beta^{\prime} \in \mathscr{A}(\Lambda)$, $\alpha_{j} \in \Pi^{i m}$ such that $\left(\Lambda \mid \alpha_{j}\right)=\left(\beta^{\prime} \mid \alpha_{j}\right)=0$. Put $\lambda:=(w, 0) \circ \Lambda=w(\Lambda+\rho)-\rho$ and $\alpha:=w\left(\alpha_{j}\right) \in W \cdot \Pi^{i m}$. Then, as before, we have an embedding

$$
T(\lambda): V(\lambda-\alpha)=V\left(\left(w, \alpha_{j}\right) \circ \Lambda\right) \hookrightarrow V((w, 0) \circ \Lambda)=V(\lambda) .
$$

Put $\mu:=\left(z, \beta_{2}\right) \circ \Lambda$ and $N(\lambda):=V(\lambda) / T(\lambda) V(\lambda-\alpha)$. Then we have

$$
[V(\lambda): L(\mu)]=[N(\lambda): L(\mu)]+[V(\lambda-\alpha): L(\mu)] .
$$

It follows from the induction hypothesis that

$$
[V(\lambda-\alpha): L(\mu)]=\left[V\left(\left(w, \alpha_{j}\right) \circ \Lambda\right): L\left(\left(z, \beta_{2}\right) \circ \Lambda\right)\right]=P_{w, z}(1),
$$

since $\operatorname{ht}\left(\beta_{2}\right)-\operatorname{ht}\left(\alpha_{j}\right)=t-1$. Thus we must show that

$$
[V(\lambda) / T(\lambda) V(\lambda-\alpha): L(\mu)]=[N(\lambda): L(\mu)]=0 .
$$

On the other hand, by Theorem 4.3.12, the module $N(\lambda)$ has Jantzen's filtration

$$
N(\lambda) \supset N(\lambda)_{1} \supset N(\lambda)_{2} \supset \cdots .
$$

Here it is obvious that

$$
\begin{aligned}
{[N(\lambda): L(\mu)] } & =\left[N(\lambda) / N(\lambda)_{1}: L(\mu)\right]+\left[N(\lambda)_{1}: L(\mu)\right] \\
& =\left[N(\lambda)_{1}: L(\mu)\right]
\end{aligned}
$$

since $N(\lambda) / N(\lambda)_{1} \cong L(\lambda)$, and $\lambda \neq \mu$. Hence what we have to show is that $\left[N(\lambda)_{1}: L(\mu)\right]=0$.

We will prove that $\sum_{i \geq 1}\left[N(\lambda)_{i}: L(\mu)\right]=0$. By Jantzen's character sum formula (4.3.1) in Theorem 4.3.12, together with Proposition 5.2.1, we have (as in the proof of Proposition 5.2.1)

$$
\begin{aligned}
\sum_{i \geq 1}\left[N(\lambda)_{i}: L(\mu)\right]= & \sum_{\beta \in \Delta_{+}} \sum_{\substack{l \in \mathbb{Z}_{\geq 1} \\
L_{l \beta}(\lambda)=0}}[V(\lambda-l \beta): L(\mu)] \\
& -\sum_{\gamma \in \Delta_{+}} \sum_{\substack{m \in \mathbb{Z}_{\geq 1} \\
L_{m \gamma}(\lambda-\alpha)=0}}[V(\lambda-\alpha-m \gamma): L(\mu)]-[V(\lambda-\alpha): L(\mu)] .
\end{aligned}
$$

Now let $\beta \in \Delta_{+}, l \in \mathbb{Z}_{\geq 1}$ be such that $L_{l \beta}(\lambda)=0$, or equivalently, such that $2(w(\Lambda+\rho) \mid \beta)=l(\beta \mid \beta)$. Then, as in the proof of Theorem 5.1.1, we have either of the following. 
Case (a): $\beta \in \Delta_{+} \cap \Delta^{r e}$ with $r_{\beta} w \geqslant w$. In this case, it follows from Theorem 3.3.3, together with the induction hypothesis, that

$$
\begin{aligned}
{[V(\lambda-l \beta): L(\mu)] } & =\left[V\left(\left(r_{\beta} w, 0\right) \circ \Lambda\right): L\left(\left(z, \beta_{2}\right) \circ \Lambda\right)\right] \\
& = \begin{cases}P_{r_{\beta}} w, z & \text { if } z \geqslant r_{\beta} w, \\
0 & \text { otherwise },\end{cases}
\end{aligned}
$$

since $z \geqslant r_{\beta} w \geqslant w$ implies $\ell(z)-\ell\left(r_{\beta} w\right) \supsetneqq \ell(z)-\ell(w)=s$ (note that $\left.r_{\beta} w \neq w\right)$.

Case (b): $l=1, \beta=w\left(\alpha_{k}\right)$ for some $\alpha_{k} \in \Pi^{i m}$ with $\left(\Lambda \mid \alpha_{k}\right)=0$. In this case, it follows again from Theorem 3.3.3, together with the induction hypothesis, that

$$
\begin{aligned}
{[V(\lambda-l \beta): L(\mu)] } & =\left[V\left(\left(w, \alpha_{k}\right) \circ \Lambda\right): L\left(\left(z, \beta_{2}\right) \circ \Lambda\right)\right] \\
& = \begin{cases}P_{w, z}(1) & \text { if } \beta_{2} \geqslant \alpha_{k}, \\
0 & \text { otherwise, }\end{cases}
\end{aligned}
$$

since $\operatorname{ht}\left(\beta_{2}\right)-\mathrm{ht}\left(\alpha_{k}\right)=t-1$.

Therefore, we deduce that

$$
\sum_{\beta \in \Delta_{+}} \sum_{\substack{l \in \mathbb{Z}_{\geq 1} \\ L_{l \beta}(\lambda)=0}}[V(\lambda-l \beta): L(\mu)]=\sum_{\substack{\beta \in A_{+} \cap \Delta^{r e} \\ z \geqslant r_{\beta} w \geqslant w}} P_{r_{\beta} w, z}(1)+\operatorname{ht}\left(\beta_{2}\right) P_{w, z}(1) .
$$

Here we remark that for fixed $z, w \in W$, the cardinality of the set $\{\beta \in$ $\left.\Delta_{+} \cap \Delta^{r e} \mid z \geqslant r_{\beta} w \geqslant w\right\}$ is finite.

Now let $\gamma \in \Delta_{+}, m \in \mathbb{Z}_{>1}$ be such that $L_{m \gamma}(\lambda-\alpha)=0$, or equivalently, such that $2\left(w\left(\Lambda+\rho-\alpha_{j}\right) \mid \gamma\right)=m(\gamma \mid \gamma)$. Then, as in the proof of Proposition 5.2.1, we have either of the following.

Case (c): $\gamma \in \Delta_{+} \cap \Delta^{r e}$ with $r_{\gamma} w \geqslant w$. In this case, it follows from Theorem 3.3.3 and the induction hypothesis that

$$
\begin{aligned}
{[V(\lambda-\alpha-m \gamma): L(\mu)] } & =\left[V\left(\left(r_{\gamma} w, \alpha_{j}\right) \circ \Lambda\right): L\left(\left(z, \beta_{2}\right) \circ \Lambda\right)\right] \\
& = \begin{cases}P_{r_{\gamma} w, z}(1) & \text { if } z \geqslant r_{\gamma} w, \\
0 & \text { otherwise, }\end{cases}
\end{aligned}
$$

since $\ell(z)-\ell\left(r_{\gamma} w\right) \supsetneqq \ell(z)-\ell(w)=s$ and $\operatorname{ht}\left(\beta_{2}\right)-\mathrm{ht}\left(\alpha_{j}\right)=t-1$.

Case (d): $m=1, \gamma=w\left(\alpha_{k}\right)$ for some $\alpha_{k} \in \Pi^{i m}$ with $\left(\Lambda-\alpha_{j} \mid \alpha_{k}\right)=0$. In this case, it follows again from Theorem 3.3.3 and the induction hypothesis that

$$
\begin{aligned}
{[V(\lambda-\alpha-m \gamma): L(\mu)] } & =\left[V\left(\left(w, \alpha_{j}+\alpha_{k}\right) \circ \Lambda\right): L\left(\left(z, \beta_{2}\right) \circ \Lambda\right)\right] \\
& = \begin{cases}P_{w, z}(1) & \text { if } \beta^{\prime} \geqslant \alpha_{k}, \\
0 & \text { otherwise },\end{cases}
\end{aligned}
$$

since ht $\left(\beta_{2}\right)-\mathrm{ht}\left(\alpha_{j}+\alpha_{k}\right)=t-2$.

Therefore, we deduce that

$$
\sum_{\gamma \in \Delta_{+}} \sum_{\substack{m \in \mathbb{Z}_{\geq 1} \\ L_{m y}(\lambda-\alpha)=0}}[V(\lambda-\alpha-m \gamma): L(\mu)]=\sum_{\substack{\gamma \in A_{+} \cap \Delta^{r e} \\ z \geqslant r_{\gamma} w \geqslant w}} P_{r_{\gamma} w, z}(1)+\mathrm{ht}\left(\beta^{\prime}\right) P_{w, z}(1) .
$$


Summarizing the above argument, we obtain that

$$
\begin{aligned}
\sum_{i \geq 1}\left[N(\lambda)_{i}: L(\mu)\right]= & \sum_{\substack{\beta \in A_{+} \cap \Delta^{r e} \\
z \geqslant r_{\beta} w \geqslant w}} P_{r_{\beta} w, z}(1)+t P_{w, z}(1) \\
& -\sum_{\substack{\gamma \in \Delta_{+} \cap \Delta^{r e} \\
z \geqslant r_{\gamma} w \geqslant w}} P_{r_{\gamma} w, z}(1)-(t-1) P_{w, z}(1)-P_{w, z}(1) \\
= & 0 .
\end{aligned}
$$

Thus, we have completely proved the theorem.

6.2. Proof of the character formula. Here we shall derive the character formula for the irreducible highest weight $\mathfrak{g}(A)$-module $L((w, \beta) \circ \Lambda)$ with $\Lambda \in P_{+}$, $(w, \beta) \in W \times \mathscr{A}(\Lambda)$ from the multiplicity formula above.

Before proceeding to the proof of the character formula, we prepare the following lemma.

Lemma 6.2.1. We use the notation of $\S 2.2$. For $\beta, \beta^{\prime} \in \mathscr{A}=\mathscr{A}(0)$, we have

$$
\sum_{\gamma \in \mathscr{A}}(-1)^{\mathrm{ht}(\gamma)-\mathrm{ht}(\beta)} Q_{\beta, \gamma}(q) P_{\gamma, \beta^{\prime}}(q)=\delta_{\beta, \beta^{\prime}} .
$$

Furthermore, for $(w, \beta),\left(w^{\prime}, \beta^{\prime}\right) \in W \times \mathscr{A}$, we have

$$
\begin{aligned}
& \sum_{(y, \gamma) \in W \times \mathscr{A}}(-1)^{(\ell(y)+\mathrm{ht}(\gamma))-(\ell(w)+\mathrm{ht}(\beta))} Q_{(w, \beta),(y, \gamma)}(q) P_{(y, \gamma),\left(w^{\prime}, \beta^{\prime}\right)}(q) \\
& \quad=\delta_{(w, \beta),\left(w^{\prime}, \beta^{\prime}\right)} .
\end{aligned}
$$

Proof. The second assertion immediately follows from the first one and the definition of the extended inverse Kazhdan-Lusztig polynomials. Hence it suffices to show the first assertion. Furthermore, we may assume that $\beta \leqslant \beta^{\prime}$. Then we have

$$
\begin{aligned}
\sum_{\gamma \in \mathscr{A}}(-1)^{\mathrm{ht}(\gamma)-\mathrm{ht}(\beta)} Q_{\beta, \gamma}(q) P_{\gamma, \beta^{\prime}}(q) & =\sum_{\substack{\gamma \in \mathscr{A} \\
\beta \leqslant \gamma \leqslant \beta^{\prime}}}(-1)^{\mathrm{ht}(\gamma-\beta)} \\
& =\sum_{\substack{\gamma \in \mathscr{A} \\
0 \leqslant \gamma \leqslant \beta^{\prime}-\beta}}(-1)^{\mathrm{ht}(\gamma)} \\
& =(1-1)^{\mathrm{ht}\left(\beta^{\prime}-\beta\right)} \\
& =\delta_{\beta, \beta^{\prime}}
\end{aligned}
$$

which proves the lemma.

Finally, we come to the main result of this paper.

Theorem 6.2.2 (character formula). Let $\mathfrak{g}(A)$ be a GKM algebra associated to a symmetrizable GGCM $A=\left(a_{i j}\right)_{i, j \in I}$ with $a_{i i} \neq 0 \quad(i \in I)$. Let $\Lambda \in P_{+}$. Then, for $(w, \beta) \in W \times \mathscr{A}(\Lambda)$, we have (equality in the algebra $\mathscr{E}$ )

$$
\operatorname{ch} V((w, \beta) \circ \Lambda)=\sum_{\left(w^{\prime}, \beta^{\prime}\right) \in W \times \mathscr{A}(\Lambda)} P_{(w, \beta),\left(w^{\prime}, \beta^{\prime}\right)}(1) \operatorname{ch} L\left(\left(w^{\prime}, \beta^{\prime}\right) \circ \Lambda\right) .
$$


Equivalently, for $(w, \beta) \in W \times \mathscr{A}(\Lambda)$, we have (again equality in the algebra $\mathscr{E})$

$$
\begin{aligned}
\operatorname{ch} L((w, \beta) \circ \Lambda)= & \sum_{\left(w^{\prime}, \beta^{\prime}\right) \in W \times \mathscr{A}(\Lambda)}(-1)^{\left(\ell\left(w^{\prime}\right)+\mathrm{ht}\left(\beta^{\prime}\right)\right)-(\ell(w)+\mathrm{ht}(\beta))} \\
& \cdot Q_{(w, \beta),\left(w^{\prime}, \beta^{\prime}\right)}(1) \operatorname{ch} V\left(\left(w^{\prime}, \beta^{\prime}\right) \circ \Lambda\right) .
\end{aligned}
$$

Proof. The first assertion follows directly from Theorem 6.1.2, by using Proposition 3.2.1, Definition 3.2.2, and Theorem 3.3.1. The second assertion immediately follows from the first one by using Lemma 6.2.1.

It is a well-known fact that $\operatorname{ch} V(\tau)=e(\tau) \cdot R^{-1}$ for $\tau \in \mathfrak{h}^{*}$, where $R:=$ $\prod_{\alpha \in \Delta_{+}}(1-e(-\alpha))^{\operatorname{mult}(\alpha)}$ (see [K, Chapter 10]). Hence, taking $(w, \beta)=(1,0) \in$ $W \times \mathscr{A}(\Lambda)$ in the above theorem, we have

$$
e(\rho) \cdot R \cdot \operatorname{ch} L(\Lambda)=\sum_{\left(w^{\prime}, \beta^{\prime}\right) \in W \times \mathscr{A}(\Lambda)}(-1)^{\ell\left(w^{\prime}\right)+\mathrm{ht}\left(\beta^{\prime}\right)} Q_{1, w^{\prime}}(1) e\left(w^{\prime}\left(\Lambda+\rho-\beta^{\prime}\right)\right) .
$$

Here it is known that $Q_{1, w^{\prime}}(1)=1$ for all $w^{\prime} \in W$.

Now recall that the set $\mathscr{A}(\Lambda)$ coincides with the set $\mathscr{S}(\Lambda)$ under the condition on the GGCM $A=\left(a_{i j}\right)_{i, j \in I}$ that $a_{i i} \neq 0 \quad(i \in I)$. Thus we have recovered the Weyl-Kac-Borcherds character formula for the irreducible highest weight $\mathfrak{g}(A)$-module $L(\Lambda)$ with highest weight $\Lambda \in P_{+}$, although the character formula for $L(\Lambda)$ itself is proved without the condition above on the GGCM $A$ (see [B1] or [K, Chapter 11]).

Remark 6.2.3. From the above argument, we see that the restriction on the GGCM $A=\left(a_{i j}\right)_{i, j \in I}$ in Theorems 6.1.2 and 6.2.2, that $a_{i i} \neq 0 \quad(i \in I)$, is essential. We do not know what the multiplicity $\left[V((w, \beta) \circ \Lambda): L\left(\left(w^{\prime}, \beta^{\prime}\right) \circ \Lambda\right)\right]$ is, for $(w, \beta),\left(w^{\prime}, \beta^{\prime}\right) \in W \times \mathscr{A}(\Lambda)$, when this restriction is removed.

\section{REFERENCES}

[B1] R. E. Borcherds, Generalized Kac-Moody algebras, J. Algebra 115 (1988), 501-512.

[B2] _ Monstrous moonshine and monstrous Lie superalgebras, Invent. Math. 109 (1992), 405-444.

[C] L. Casian, Kazhdan-Lusztig multiplicity formulas for Kac-Moody algebras, C. R. Acad. Sci. Paris 310 (1990), 333-337.

[DGK] V. V. Deodhar, O. Gabber, and V. G. Kac, Structure of some categories of representations of infinite-dimensional Lie algebras, Adv. Math. 45 (1982), 92-116.

[GW] R. Goodman and N. R. Wallach, Whittaker vectors and conical vectors, J. Funct. Anal. 39 (1980), 199-279.

[J] J. C. Jantzen, Moduln mit einem Höchsten Gewicht, Lecture Notes in Math., vol. 750, Springer, Berlin and New York, 1979.

[K] V. G. Kac, Infinite dimensional Lie algebras, 3rd ed., Cambridge Univ. Press, Cambridge, 1990.

[KK] V. G. Kac and D. A. Kazhdan, Structure of representations with highest weight of infinite-dimensional Lie algebras, Adv. Math. 34 (1979), 97-108.

[Ka] M. Kashiwara, Kazhdan-Lusztig conjecture for a symmetrizable Kac-Moody Lie algebra, The Grothendieck Festschrift, II (P. Cartier et al., eds.), Progress in Math., vol. 87, Birkhäuser, Boston, 1990, pp. 407-433. 
[KT] M. Kashiwara and T. Tanisaki, Kazhdan-Lusztig conjecture for symmetrizable Kac-Moody Lie algebra. II: Intersection cohomologies of Schubert varieties, Operator Algebras, Unitary Representations, Enveloping Algebras, and Invariant Theory (A. Connes et al., eds.), Progress in Math., vol. 92, Birkhäuser, Boston, 1990, pp. 159-195.

[KL1] D. A. Kazhdan and G. Lusztig, Representations of Coxeter groups and Hecke algebras, Invent. Math. 53 (1979), 165-184.

[KL2] _ Schubert varieties and Poincaré duality, Proc. Sympos. Pure Math., vol. 36, Amer. Math. Soc., Providence, RI, 1980, pp. 185-203.

[N] S. Naito, Kazhdan-Lusztig conjecture for generalized Kac-Moody algebras. I: Towards the conjecture, Comm. Algebra 23 (1995), 703-736.

[RW] A. Rocha-Caridi and N. R. Wallach, Highest weight modules over graded Lie algebras: Resolutions, filtrations and character formulas, Trans. Amer. Math. Soc. 277 (1983), 133-162.

[S] N. N. Shapovalov, On a bilinear form on the universal enveloping algebra of a complex semisimple Lie algebra, Functional Anal. Appl. 6 (1972), 307-312.

[W] N. R. Wallach, A class of non-standard modules for affine Lie algebras, Math. Z. 196 (1987), 303-313.

Department of Mathematics, Shizuoka University, 836 Ohya, Shizuoka 422, Japan

E-mail address: smsnait@sci.shizuoka.ac.jp 